\title{
Development of consonance preferences in Western listeners
}

\author{
Michael W. Weiss, Laura K. Cirelli, Josh H. McDermott, and \\ Sandra E. Trehub
}

Version Post-print/Accepted Manuscript

Citation Weiss, M. W., Cirelli, L. K., McDermott, J. H., \& Trehub, S. E. (2020).

(published version) Development of consonance preferences in Western listeners. Journal of Experimental Psychology: General, 149(4), 634-649.

https://doi.org/10.1037/xge0000680

Copyright / License (C) 2020, American Psychological Association

Publisher's Statement This article may not exactly replicate the authoritative document published in the APA journal. It is not the copy of record. The final article is available via its DOI: https://doi.org/10.1037/xge0000680. 
Development of consonance preferences in Western listeners

Michael W. Weiss ${ }^{1,2}$, Laura K. Cirelli ${ }^{3}$, Josh H. McDermott ${ }^{4}$, and Sandra E. Trehub ${ }^{3}$

1 - Université de Montréal

2 - International Laboratory for Brain, Music, and Sound Research

3 - University of Toronto

4 - Massachusetts Institute of Technology

Author Note

Funded by the Natural Sciences and Engineering Research Council of Canada.

Preliminary results were presented at the meeting of the Society for Music Perception and

Cognition (San Diego, CA, 2017). Address correspondence to Michael W. Weiss, Department of

Psychology, University of Montreal, CP 6128, succ. Centre-ville, Montreal, QC, H3C 3J7,

Canada. Email at michael.william.weiss@gmail.com 


\begin{abstract}
Many scholars consider preferences for consonance, as defined by Western music theorists, to be based primarily on biological factors, while others emphasize experiential factors, notably the nature of musical exposure. Cross-cultural experiments suggest that consonance preferences are shaped by musical experience, implying that preferences should emerge or become stronger over development for individuals in Western cultures. However, little is known about this developmental trajectory. We measured preferences for the consonance of simultaneous sounds and related acoustic properties in children and adults to characterize their developmental course and dependence on musical experience. In Study 1, adults and children 6 to 10 years of age rated their liking of simultaneous tone combinations (dyads) and affective vocalizations. Preferences for consonance increased with age and were predicted by changing preferences for harmonicity the degree to which a sound's frequencies are multiples of a common fundamental frequency but not by evaluations of beating - fluctuations in amplitude that occur when frequencies are close but not identical, producing the sensation of acoustic roughness. In Study 2, musically trained adults and 10-year-old children also rated the same stimuli. Age and musical training were associated with enhanced preference for consonance. Both measures of experience were associated with an enhanced preference for harmonicity, but were unrelated to evaluations of beating stimuli. The findings are consistent with cross-cultural evidence and the effects of musicianship in Western adults in linking Western musical experience to preferences for consonance and harmonicity.
\end{abstract}

Keywords: consonance, development, aesthetic preferences, music 
Development of consonance preferences in Western listeners

Western adults' evaluative judgments of simultaneously sounding tones are relatively consistent. They prefer tone combinations considered consonant, according to Western music conventions, to those considered dissonant (e.g., Oxenham, 2013). Nevertheless, there are divergent perspectives on consonance and dissonance both within the disciplines of music and science and across those disciplines, some emphasizing biological factors and others emphasizing experiential factors (Parncutt \& Hair, 2011; Tramo, Cariani, Delgutte, \& Braida, 2001; Zentner \& Kagan, 1996). Musical intervals designated as consonant or dissonant in Western music have changed over historical periods, as reflected in compositional and performance practices (Cazden, 1980; Tenney, 1988). Moreover, ethnomusicologists have documented the prominence of so-called dissonances in musical systems around the world (e.g., Tenzer, 1991; Jordania, 2006), highlighting the possibility of cultural and experiential contributions to aesthetic judgments. There are three natural sources of evidence for the effects of experience: cross-cultural studies, developmental studies on infants or children, and comparisons of listeners with different degrees of musical experience. The primary goal of the current research was to document listeners' evaluative judgments of tone combinations as a function of age and musical experience. Naturally, such experiential contributions do not rule out important biological contributions to aspects of musical structure and perception.

Recent research supports the importance of experience, incidental or deliberate, in shaping aesthetic judgments of consonant and dissonant stimuli (McDermott, Schultz, Undurraga, \& Godoy, 2016; McLachlan, Marco, Light, \& Wilson, 2013). For instance, consonance preferences are stronger in musicians than in non-musicians (McDermott, Lehr, \& 
Oxenham, 2010; Dellacherie, Roy, Hugueville, Peretz, \& Samson, 2011). Such preferences can also be altered by short-term laboratory experience (McLachlan et al. 2013).

Cross-cultural evidence highlights the role of experience in preferences for consonant stimuli. The widespread use of a subset of consonant simultaneous intervals (octaves, perfect fourths, perfect fifths) in music across cultures (Brown \& Jordania, 2013; Savage, Brown, Sakai, \& Currie, 2015) has led some to argue for biological constraints favoring canonically consonant intervals (Schellenberg \& Trehub, 1996; Purves, 2017). However, such constraints need not apply to aesthetic responses. For instance, musical systems may exhibit pitch intervals with simple integer ratios simply because they are easy to produce on simple musical instruments, and listeners might develop preferences for those intervals from incidental musical exposure. Ethnomusicologists have noted the prominence of dissonant intervals (Jordania, 2006; Vassilakis, 2005) or tuning systems (Vetter, 1989) in some musical cultures, indicating that any such constraints are not absolute. Moreover, it is not obvious that the use of particular intervals in a musical system necessitates positive evaluations of those intervals. Definitive cross-cultural evidence for the basis of consonance preferences thus requires evaluative judgments by listeners with minimal exposure to Western music. In the only study of that nature, the Tsimane', an isolated Amazonian community with little exposure to Western music or any kind of polyphony, exhibited no preference for consonant over dissonant simultaneous intervals, despite exhibiting preferences for other acoustic contrasts (McDermott et al., 2016). For Bolivians in nearby towns and cities, consonance preferences were graded, in line with their presumptive exposure to Western music. These results are therefore suggestive of an aesthetic response that emerges from experience with music featuring consonant pitch combinations. 
Development (i.e., age-related change) provides another natural source of evidence for the role of musical experience. Perceptual sensitivity to harmonicity is present by 4 months of age (Folland, Butler, Payne, \& Trainor, 2015; He \& Trainor, 2009), although it improves with age. Other musically relevant skills are shaped by culture-specific musical experience. For example, cultural biases in rhythm discrimination are prominent in adults (Jacoby \& McDermott, 2017) and are evident by 12 months of age (Hannon \& Trehub, 2005a, b). Listening biases for culture-specific rhythms emerge even earlier (Soley \& Hannon, 2010). Enculturation to culturespecific pitch patterns has a longer developmental trajectory. For example, sensitivity to Western key structure and harmony emerges in the preschool years (Corrigall \& Trainor, 2014; Kragness \& Trainor, 2018), an achievement that is accelerated by early musical training (Corrigall \& Trainor, 2010). Collectively, these studies show that children possess a relatively flexible template for musical conventions, which could extend to consonance.

Infants also exhibit differential responsiveness to consonant and dissonant tone combinations. After 9-month-olds are exposed to repeating pure-tone dyads separated by 5, 6, or 7 semitones (highly consonant, highly dissonant, and highly consonant, respectively), they readily detect subtle frequency differences in the context of the consonant combinations but not the dissonant combination, indicating differences in the processing of consonant and dissonant harmonic and melodic intervals (Schellenberg \& Trehub, 1996). Problems arise, however, with attempts to interpret infant processing differences as aesthetic preferences. In a number of studies involving brief listening tasks, newborns and older infants listened longer to a musical excerpt with consonant intervals than to one with dissonant intervals (Masataka, 2006; Trainor \& Heinmiller, 1998; Zentner \& Kagan, 1996). Listening biases were more ambiguous when 2- to 4month-olds were tested with isolated (repeating) chords (Trainor, Tsang, \& Cheung, 2002). Such 
evidence of biased listening was interpreted as reflecting early, likely innate, aesthetic preferences for consonance. However, similar test procedures in other studies with infants have revealed listening biases for familiar stimuli in some cases and for novel stimuli in others (Houston-Price \& Nakai, 2004). Moreover, when 6-month-olds receive 3 min of exposure to consonant or dissonant patterns prior to their listening "preference" test, they listen longer to the previously heard stimulus, whether consonant or dissonant (Plantinga \& Trehub, 2014), suggesting that their responses are driven by familiarity or ease of processing rather than aesthetic preferences. Overall, the evidence in infants is equivocal with respect to the question of innate preferences for consonance.

There has been relatively little study of children's musical preferences beyond the preschool period, when they can provide reliable aesthetic judgments. Over a century ago, Valentine (1913) asked 6- to 13-year-old children and adults to rate their liking of a series of tone combinations (dyads) that he played on a piano. He found no preference for consonant intervals in 6- or 7-year-olds, a "great advance" at 9 years of age, and adult-like preferences at 12 or 13 years of age. Musical training accelerated children's progress toward adult preferences. However, older children and adults judged the tritone (6-semitone difference) and minor sixth (8 semitones) more favorably than the perfect fifth (7 semitones), in stark contrast to contemporary Western adults. Interpretation of Valentine's study is limited by methodological problems, most notably the lack of fine stimulus control, and sparse details. Nevertheless, his findings are consistent with the gradual emergence of aesthetic preferences for consonance in an era that predated the ease of access to music.

To further test the effects of exposure to Western music on consonance preferences, we sought to assess preferences for consonance and associated acoustic factors in contemporary 
Western children of different ages, with or without musical training. The primary goal was to ascertain the age at which children's preferences for simultaneous tone combinations approximate those of adults, which has not been studied in the modern era and is relevant to current debates about preferences for consonance. We expected aesthetic judgments to change from early childhood to later childhood and adulthood and to be influenced by musical training. Although 6-year-olds, corresponding to the youngest children in the study, exhibit some sensitivity to pitch structure in Western music (Cuddy \& Badertscher, 1987), their implicit knowledge of harmony remains incomplete for some years (Krumhansl \& Keil, 1982). As a result, it was unclear whether a preference for consonant tone combinations would be evident in the youngest group.

A secondary goal was to investigate the acoustic basis of aesthetic preferences for consonance across development. Debates over the basis of consonance and dissonance have also encompassed the role of underlying acoustic phenomena, primarily beating and harmonicity. The beating account of sensory dissonance holds that unpleasant sensations arise from the frequencies of simultaneous tones being close to one another (i.e., within the same auditory filter or critical band of the basilar membrane), resulting in rapid amplitude fluctuations and the perception of beating or roughness ${ }^{1}$ (e.g. Helmholtz, 1863; Terhardt, 1974; Hutchinson \& Knopoff, 1978; Tramo, Cariani, \& Delgutte, 2001; Sethares, 2005). Visualizations of beating are provided in Figure 1A. Intervals with fundamental frequencies related by small-integer ratios, such as the octave $(2: 1)$ or perfect fifth $(3: 2)$, have harmonics that coincide or are widely spaced and are therefore relatively free of beats or roughness. By contrast, intervals with fundamental

\footnotetext{
${ }^{1}$ Technically, roughness is a perceptual attribute related to beating but constrained by the critical bandwidths of the ear (Terhardt, 1974).
} 
frequencies related by large-integer ratios, such as the tritone $(45: 32)$, have harmonics that interact within the same critical band, usually resulting in perceptible beats.

Although there is consensus about the factors that generate the perception of beating, there are reasons to doubt the proposed link between beating and consonance judgments. For example, preferences for non-beating stimuli are robust even in listeners with impaired pitch perception who do not exhibit consonance preferences (Cousineau, McDermott, \& Peretz, 2012). Moreover, when pure-tone dyads are presented dichotically (i.e., in separate ears) to preclude beating, listeners still evaluate dissonant intervals negatively, although their dislike is attenuated for smaller intervals (i.e., 1-3 semitones; McDermott et al., 2010). In other words, beating decreases pleasantness, but it fails to account for listeners' preferences for some intervals over others.

The harmonicity account of consonance (e.g. McDermott et al., 2010; Stumpf, 1890; Tramo, Cariani, \& Delgutte, 2001) instead holds that the favorability of a tone combination is influenced by the degree to which the combined frequencies from two or more simultaneous tones resemble those of the harmonic series. Visualizations of harmonicity and inharmonicity are provided in Figures 1B-C. Pitched sounds, as produced by voices or instruments, have energy at the fundamental frequency and at integer multiples (harmonics) of this frequency. The auditory system uses this harmonic information to identify sound sources in complex sound environments (Bregman, 1990). For instance, human listeners detect when a single harmonic is mistuned in a speech signal (Popham et al., 2018) or synthetic tone (Moore et al. 1986; Folland, Butler, Smith, \& Trainor, 2012; Alain, Arnott, \& Picton, 2001), hearing it as coming from a separate source. Listeners are thus highly sensitive to whether or not sounds are harmonic. Harmonicity is predictive of consonance because note combinations that are consonant to Westerner listeners 
produce aggregate frequency spectra that are approximately harmonic, whereas canonically dissonant intervals do not (Figure 1B versus 1C).

It is difficult to assess the separate contributions of beating and harmonicity when natural stimuli are used because intervals with large-integer ratios tend to be both rough and inharmonic whereas those with small-integer ratios are less rough as well as harmonic (see Figures 1B-E). With artificial stimuli, however, beating and harmonicity can be manipulated independently (Cousineau et al., 2012; McDermott et al., 2010, 2016). These studies revealed that although listeners prefer stimuli that lack beating, their preference for non-beating stimuli does not correlate with their preference for consonant intervals. By contrast, listeners' preference for harmonic tones is correlated with their preference for consonant intervals, both within and across cultures (McDermott et al., 2010; McDermott et al., 2016). Acoustic preferences also provide evidence for experiential contributions to consonance preferences, in that preferences for harmonicity and consonance correlate with years of musical training, while preferences for nonbeating stimuli do not (McDermott et al., 2010). Thus, even though harmonicity-based sound segregation is believed to emerge in typical human environments regardless of musical experience, perhaps because it is critical for speech perception and voice recognition (McPherson \& McDermott, 2018), the aesthetic response to harmonicity varies across Western listeners in rough proportion to their musical experience.

The second goal of the present study was thus to further investigate this issue by measuring preferences related to harmonicity and beating over development. In line with the studies above, we expected harmonicity to have a greater influence on consonance preferences than beating, and for harmonicity preferences to increase with age and musical training. 
In Study 1, adults and 6-, 8-, and 10-year-old children rated their liking of musical intervals in two timbres (synthesizer, voice) as well as artificial sounds that isolate the acoustic features of harmonicity and beating, stimuli used previously with Western and non-Western adults (McDermott et al., 2016). In addition, a set of positive and negative non-musical vocalizations were used to ensure that children were capable of using the rating scale. In Study 2, we used the same method to examine the effect of music lessons on consonance preferences in 10-year-old children and adults.

\section{Study 1}

We compared evaluative ratings for tone combinations in children and adults with limited musical training.

\section{Method}

Participants. The participants were $6(n=23,11$ female, $M=6.4, S D=0.3$, range $=6.1-$ 7.4 years $), 8(n=25,12$ female, $M=8.4, S D=0.2$, range $=8.1-8.8$ years $), 10(n=23,12$ female, $M=10.3, S D=0.2$, range $=10.0-11.2$ years $)$, and 20 years of age $(n=26,14$ female, $M$ $=20.0, S D=2.6$, range $=17.9-26.8$ years $)$, all of whom were born in Canada. Sample sizes were determined using effect sizes and variability reported in previous research with participants who varied in musical experience (McDermott et al., 2016). Children had a few months of music lessons on average, a maximum of 2 years $(M=0.3, S D=0.5$, range $=0-2$ years $)$, and no ongoing training. Adults had one year of music lessons on average, a maximum of 3 years of lessons, and no current lessons or regular musical practice $(M=1.0, S D=1.0$, range $=0-3$ years). An additional four children were excluded because of illness on the day of testing $(n=1)$, failure to follow the test protocol $(n=2)$, or failure to complete all trial stimulus sets $(n=1)$. An additional 12 children and 1 adult were excluded because of perseverative responding in one or 
more trial stimulus sets. Perseverative responding was defined as a run of repeated responses exceeding two standard deviations from the mean of the length of the longest run of repeated responses for each child, calculated separately for each stimulus set. This research was approved by the Human Research Ethics Program of the University of Toronto.

Stimuli. The stimuli, listed below, were identical to those used in previous research (McDermott et al., 2010, 2016) except as noted. All stimuli were normalized to the same rootmean-square (RMS) level, and were presented at a comfortable listening level (approximately 60 dB SPL, C weighted). Sample audio stimuli are available as supplemental materials and the full set of stimuli is available by request.

Classification of the relative consonance of intervals can differ across cultures and eras. For the present purposes intervals were defined as consonant or dissonant according to ratings provided by American adults in previous research with similar stimuli (McDermott et al., 2010). That study was used as baseline because it included a large sample from a similar population (i.e., Americans) who were tested recently with similar stimuli (i.e., synthetic and sung two-tone dyads). As seen in Table 1, five intervals were considered dissonant (min2, maj2, tritone, min7, maj7) and seven were considered consonant (min3, maj3, perf4, perf5, min6, maj6, octave). Minor changes in classification (e.g., considering min6 as dissonant) had no appreciable effect on results.

Vocalizations (use of rating scale). To assess the use of the rating scale, participants were presented with 5 exemplars of 3 types of non-musical emotional vocalizations (happy, sad, fearful) taken from the Montreal Affective Voices set (Belin, Fillion-Bilodeau, \& Gosselin, $2008)$, for a total of 15 trials. Vocalizations varied in duration $(M=1.5, S D=1.0$, range $=0.3-$ $4.3 \mathrm{~s}$ ) and were recorded as $16 \mathrm{bit} / 44.1 \mathrm{kHz}$ audio. 
Synthetic intervals. Synthetic intervals were composed of complex tones with 10 harmonics of decreasing amplitude (14 dB/octave), a percussive amplitude envelope (i.e., 10-ms half-Hanning window attack, $2.5 \mathrm{~s}^{-1}$ exponential decay), and an overall duration of $2 \mathrm{~s}$, generated as $16 \mathrm{bit} / 20 \mathrm{kHz}$ audio. There were 13 intervals (unison to octave) with just intonation, each with 4 exemplars (differing in root pitch, set to $\mathrm{C} \# 4$, D4, D\#4, or E4, with $\mathrm{C} 4$ as middle C), for a total of 52 trials. These were identical to the stimuli of McDermott et al. (2010) apart from being in just intonation rather than equal temperament.

Sung intervals. Sung intervals were recorded by a professional singer who pitch-matched to the equal-tempered scale. The amplitude envelope of each note was modified such that the onset ramped up from silence (i.e., 10-ms half-Hanning window), and the last half of each note faded linearly to zero, with an overall duration of $2 \mathrm{~s}$, recorded as $16 \mathrm{bit} / 20 \mathrm{kHz}$ audio. Sung notes were assembled into intervals with equal temperament to the extent achievable by the singer. The stimulus set consisted of 4 exemplars (root pitch G\#3, A4, A\#4, or B4) of each of 12 intervals (unison to major seventh; the octave was omitted because it stretched the range of the singer), for a total of 48 trials. Half of the stimuli (root pitches G\#3 and A\#4) were sung with the vowel 'ooh', and the other half with 'aah'. These stimuli were taken from those used in McDermott et al. (2010).

Beating and non-beating tone-pairs. Rough stimuli (i.e., producing audible beating) were generated by synthesizing two tones that were similar but not identical in frequency and presenting them diotically. A smooth (i.e., non-beating) version of each was created by presenting the same tone pairs dichotically. Dichotic presentation prevents the component frequencies from interacting within the peripheral auditory system, and thus largely eliminates audible beating (Helmholtz, 1863; Rutschmann \& Rubinstein, 1965; McDermott et al., 2010). 
Cochlear nonlinearity could have produced distortion products that in principle might also have differentiated diotic and dichotic presentation. In practice, however, distortion products are substantially lower in level than the stimulus frequencies that enter the ear (Pressnitzer \& Patterson, 2001; Norman-Haignere \& McDermott, 2016), and are thus unlikely to substantially affect the evaluation of the stimulus. This assumption is supported by the finding that the difference in pleasantness between diotic and dichotic presentation is present only for intervals less than a major third (which produce beating in the diotic case, presumably because they fall within the same cochlear filter) (McDermott et al., 2010), even though distortion products would be produced for larger intervals as well.

The difference between the root frequency and the second frequency was 1.5 semitones for low and mid stimuli, and 0.75 semitones for high stimuli, selected to produce salient beating and roughness. The beat frequency is the difference between the component frequencies, and increases with register for a given interval in semitones. A smaller interval was thus chosen for the highest register. Each type of tone pair (smooth/dichotic, rough/diotic) was presented at three different registers, separated by octaves (low [C4], mid [C5], or high [C6]), and at four root pitches in the assigned octave (C, C\#, D, D\#), yielding 24 trials. Amplitude envelopes were the same as in the synthetic interval set.

Harmonic and inharmonic stimuli. To assess the effect of harmonicity, eight harmonic stimuli were generated with a fundamental frequency $(C 4, C \# 4, D 4$, or D\#4) and two subsets of harmonics of the fundamental $(1,2,4$, and 8 , or 1, 2, 3, 5, and 9). Numerically adjacent harmonics were presented to different ears (i.e., left ear $[1,4]$ and right ear $[2,8]$; left ear $[1,3,5$, 9] and right ear [2]). Eight inharmonic stimuli were generated by perturbing the frequencies of the harmonic stimuli. Four of the inharmonic stimuli were 'jittered', with harmonics 2 and 5 
presented in one ear and increased by 0.5 semitones, and harmonics 1,3 , and 9 presented in the other ear and decreased by 0.5 semitones. The other four inharmonic stimuli were 'shifted', with each harmonic $(1,2,3,5$ and 9$)$ increased in frequency by $30 \mathrm{~Hz}$. The fundamental frequency used to generate the shifted stimuli was decreased by the shift amount so that the frequency of the lowest component remained constant (the frequency of each component was (f0 - 30)* $\mathrm{h}+30$, where h was the harmonic number). Shifted stimuli were presented with even and odd harmonics in different ears. Even and odd harmonics were presented to different ears to minimize any differences in beating between the harmonic and inharmonic stimuli, such that they would differ primarily in harmonicity.

In addition to the eight harmonic and eight inharmonic stimuli described above, four pure-tone stimuli with frequencies set to the fundamental frequencies of the harmonic stimuli (i.e., $\mathrm{C} 4, \mathrm{C \# 4}$, D4, or D\#4) were included in the calculation of a score for harmonic stimuli (i.e., $n=12$ harmonic trials total) and four smooth/non-beating trials (i.e., low pitch + dichotic) were included in the calculation of a score for inharmonic stimuli (i.e., $n=12$ inharmonic trials total), following the method in McDermott et al. (2016). For all stimuli, amplitude envelopes were the same as in the synthetic interval set.

Apparatus. Participants were tested individually in a double-walled sound-attenuating booth (Industrial Acoustics, Inc.). Stimuli were presented over Sony MDR-7506 headphones using a custom presentation program written in Matlab (R2016a) and PsychToolBox (3.0.13) and implemented on an iMac computer (Apple, Inc.).

Procedure. Children were tested in the presence of an experimenter to ensure their comfort and compliance. The experimenter could hear the stimuli (via headphones) only during the practice trials. At the beginning of each session, the experimenter explained the trial format 
to the child: a trial was initiated when the child indicated readiness, the stimulus was presented, and four visual response options appeared on the screen (see Figure 2). The experimenter described the various facial expressions as "really don't like it", "don't like it a little", "like it a little", and "really like it". This procedure was adapted from a paradigm previously used crossculturally in adults (McDermott et al., 2016). In both cases we chose to have participants assess "liking" with a discrete set of verbal labels rather than "pleasantness" using a numerical rating scale (as in some other prior work on consonance, e.g., McDermott et al., 2010) to maximize the likelihood of comprehension across different participant groups. In some cases "liking" and "pleasantness" may be dissociable (Ritossa \& Rickard, 2004), but we have found them to yield indistinguishable results for interval/chord evaluation. While the notion of consonance is distinct from that of pleasantness in listeners with considerable musical education (e.g., degrees in music), liking or pleasantness judgments appear to be synonymous with consonance in musically untrained individuals, such as those tested here (Arthurs et al., 2018). Children were asked to point to the face that "matches how much you liked that sound," and the experimenter recorded their choice via a mouse click. For deviations in protocol during a trial, which occurred infrequently (on $0.1 \%$ of trials), the experimenter could select an option to "pass" without providing a response, and the trial was lost.

The session began with five practice trials, after which the experimenter presented each of the stimulus sets in random order. Each stimulus set contained one stimulus category except for the harmonicity and beating stimuli, which were presented in the same set. After each set, children received a Canadian two-dollar coin, which they placed in a personalized envelope to maintain their motivation for the next stimulus set. The envelope was placed out of sight before the next set began. Short breaks were permitted between sets. Test sessions lasted about $30 \mathrm{~min}$. 
The test protocol was identical for adult participants except for the absence of the experimenter and coin dispensing between stimulus sets.

\section{Results}

Because preferences for consonant versus dissonant intervals were hypothesized to reflect cumulative exposure to music, age group was considered as a continuous, equally-spaced covariate (i.e., 1, 2, 3, 4), which preserved the ordinal nature of the grouping variable. The predictor was mean-centered to prevent distortion of within-subject effects (Schneider, AviviReich, \& Mozuraitis, 2015).

Use of rating scale. The vocalization set was included to assess age-related differences in the use of the rating scale and general aesthetic preferences, on the assumption that valence associations with the vocalizations would be independent of musical experience. Ratings were averaged according to whether the trial featured a positive vocalization ( $n=5$ happy trials) or negative vocalization ( $n=10$ sad or fearful trials). Descriptive statistics are visualized in Figure 3A. A repeated-measures ANCOVA compared mean ratings, with vocalization type (positive, negative) as a within-participant factor and age group as a covariate. As expected, there was a robust main effect of vocalization type, $F(1,95)=147.16, p<.001, \eta_{p}{ }^{2}=0.61$, with lower ratings for negative stimuli $(M=1.97, S D=0.73)$ than for positive stimuli $(M=3.08, S D=$ 0.61). There was also a main effect of age group, $F(1,95)=19.64, p<.001, \eta_{p}{ }^{2}=.17$, driven by a negative correlation between age group and overall ratings, $r(95)=-.41, p<.001$. Importantly, there was no interaction between age group and stimulus valence, $F<1$, confirming that participants across age used the rating scale in a similar manner to differentiate negative from positive stimuli. 
Preference for consonance. Ratings from the two stimulus sets that presented note dyads (synthetic, sung) were averaged according to consonance or dissonance grouping (see Stimuli; Table 1), yielding four scores per participant (see Figures 3B-C). The number of trials averaged per score differed slightly across sets due to differences in the range of intervals (see Stimuli). Figure 4 displays descriptive statistics for ratings by individual intervals across stimulus sets and age groups.

A repeated-measures ANCOVA examined ratings as a function of the within-subject factors of consonance (consonant, dissonant) and stimulus set (synthetic, sung), with age group considered as a covariate in the model. There was a significant main effect of age, $F(1,95)=$ $13.04, p<.001, \eta_{p}^{2}=.12$, reflecting a tendency for ratings to decrease with age, $r(95)=-.35, p<$ .001 , as was also observed in the vocalization set. There was an interaction between age and stimulus set, $F(1,95)=9.91, p=.002, \eta_{p}{ }^{2}=.09$. A series of correlations (Holm-Bonferroni corrected) showed that overall ratings did not correlate with age for the sung stimulus set, $p>.1$, but declined with age in the synthetic stimulus set, $r(95)=-.44, p<.001$. There were no other effects or interactions involving stimulus set, $p \mathrm{~s}>.2$. The main effect of consonance was significant, $F(1,95)=96.95, p<.001, \eta_{p}^{2}=.51$, and was qualified by a significant interaction between age and consonance, $F(1,95)=6.40, p=.013, \eta_{p}{ }^{2}=.06$.

The interaction between age and consonance level was due to an increasing preference for consonance with age, $r(95)=.25, p=.013$. The interaction was explored by correlating age group with ratings for only consonant or only dissonant stimuli (i.e., averaged across stimulus sets). There was a negative correlation between age group and ratings for consonant stimuli, $r(95)=-.27, p=.007$, and a stronger negative correlation between age group and ratings for dissonant stimuli, $r(95)=-.37, p<.001$ 
An interaction between age group and consonance could be present even if some age groups did not exhibit a preference for consonance. A series of paired-samples $t$-tests (HolmBonferroni corrected), within each age group and within each stimulus set, compared ratings for consonant versus dissonant intervals. Despite the reduction in power from dividing the sample, ratings were significantly higher for consonant than for dissonant stimuli in each subtest, $p \mathrm{~s}<$ .02 , which confirms that the interaction in the ANCOVA represents sharpening of the consonance preference, which was present to some extent in all groups.

The development of the preference for consonance could also be framed relative to adults rather than chance. In other words, at what age does preference for consonance become adultlike? A series of three independent samples $t$-tests (Holm-Bonferroni corrected) compared overall preference for consonance (i.e., collapsed across stimulus set) between adults and each age group of children. Preference for consonance did not differ between adults $(M=0.46, S D=$ $0.31)$ and 10-year-olds $(M=0.36, S D=0.41), t(47)=0.98, p>.3$, Cohen's $d=0.28$, but it did differ between adults and 8-year-olds $(M=0.25, S D=0.28), t(49)=2.51, p=.046$, Cohen's $d=$ 0.70 , and between adults and 6-year-olds $(M=0.25, S D=0.32), t(47)=2.37, p=.046$, Cohen's $d=0.68$. It is likely that differences between adults and children aged 10 (or older) could be observed with more statistical power (see also Study 2), but the effect size would likely diminish as the age of the comparison group increased. In any case, the current data suggest that an adultlike preference for consonance does not emerge until at least 10 years of age.

Small and large dissonant intervals. It appears from Figure 4 that the sharpening of consonance preferences with age is driven by some intervals more than others. To assess any such effects, a repeated-measures ANCOVA compared ratings by interval size (small, large), with age group as a covariate. Four scores were created for each participant for the synthetic and 
sung interval sets, with ratings averaged by interval size (small: 1, 2; large: 6, 10, 11 semitones). As expected from the previous tests, there was a main effect of age (Synthetic: $F(1,95)=27.52$, $p<.001, \eta_{p}{ }^{2}=.23$; Sung: $\left.F(1,95)=4.75, p=.032, \eta_{p}{ }^{2}=.05\right)$, which confirmed the general decrease in ratings. There was also a significant main effect of interval size (Synthetic: $F(1,95)$ $=50.44, p<.001, \eta_{p}{ }^{2}=.35$; Sung: $\left.F(1,95)=26.02, p<.001, \eta_{p}{ }^{2}=.22\right)$. More notably, the main effects were qualified by an interaction between age and interval size (Synthetic: $F(1,95)=$ 12.11, $p<.001, \eta_{p}{ }^{2}=.11$; Sung: $\left.F(1,95)=5.78, p=.018, \eta_{p}{ }^{2}=.06\right)$, which was driven by a decrease in the ratings of the small intervals with age. There was a significant negative correlation between age and ratings for small dissonant intervals in both stimulus sets (Synthetic: $r(95)=-.52, p<.001$; Sung: $r(95)=-.27, p=.007)$, but a reduced or non-significant correlation between age and ratings for large dissonant intervals (Synthetic: $r(95)=-.34, p<.001$; Sung: $r(95)=-.12, p=.24)$. In short, the age-related effect in preference for consonance was driven by changing evaluation of smaller dissonant intervals.

Aversion to beating and inharmonicity. To evaluate responses to beating, two scores were calculated by averaging ratings for tone-pair trials according to presentation type (diotic, dichotic), with 12 trials per score. Descriptive statistics are shown in Figure 3D. A repeatedmeasures ANCOVA compared ratings with presentation type as a repeated factor and age group as a covariate. There were significant main effects of presentation type, $F(1,95)=100.53, p<$ $.001, \eta_{p}{ }^{2}=.51$, and age group, $F(1,95)=22.96, p<.001, \eta_{p}{ }^{2}=.19$, which were qualified by a significant interaction between age and presentation type, $F(1,95)=7.21, p=.009, \eta_{p}{ }^{2}=.07$. Two follow-up correlations revealed that the negative relationship between age group and ratings was stronger with diotic presentation, $r(95)=-.46, p<.001$, than with dichotic presentation, $r(95)=-.34, p<.001$. In other words, the advantage for dichotic presentation (i.e., dichotic 
minus diotic scores) increased with age, $r(95)=.27, p=.009$, representing an increase in the aversion to beating. Nevertheless, within each age group there were significantly lower ratings for diotic than dichotic trials, $p \mathrm{~s} \leq .005$ (Holm-Bonferroni corrected), reflecting aversion to beating even in the youngest group.

Harmonicity preferences were similarly assessed by calculating two scores per participant, one combining eight harmonic trials and four pure-tone trials (hereafter harmonic score) and another combining eight inharmonic trials and four low-diotic tone-pair trials (hereafter inharmonic score), as in McDermott et al. (2016). Descriptive statistics are shown in Figure 3E. A repeated-measures ANCOVA comparing ratings, with harmonicity as a repeated factor and age group as a covariate, revealed a significant main effect of harmonicity, $F(1,95)=$ $65.57, p<.001, \eta_{p}{ }^{2}=.41$, no main effect of age group, $F(1,95)=2.66, p=.11$, and a significant interaction between age and harmonicity, $F(1,95)=4.79, p=.031, \eta_{p}{ }^{2}=.05$. The difference between ratings for harmonic versus inharmonic stimuli correlated positively with age group, $r(95)=.22, p=.031$. Two follow-up correlations to further explore the interaction revealed that age group did not correlate with ratings for harmonic stimuli, $r(95)=-.05, p=.62$, but there was a significant negative correlation between age and ratings for inharmonic stimuli, $r(95)=-.23, p$ $=.022$. In separate paired-samples $t$-tests for each age group (Holm-Bonferroni corrected), there was an advantage for harmonic stimuli at each age, $p \mathrm{~s} \leq .012$. These results suggest that preferences for harmonicity were apparent at a young age, and that developmental changes in preferences were driven primarily by an increasing distaste for inharmonic stimuli.

The relationship between age and preference for consonance could be partially or fully explained by preference for non-beating stimuli, preference for harmonicity, or both, because age correlated with both variables in the aforementioned analyses. A multiple mediation analysis 
using the bootstrap approach (Preacher \& Hayes, 2008; 5,000 resamples) revealed that age affected preference for consonance as a function of its relationship with preference for harmonicity, $a_{1} b_{1}=0.038, S E=0.018,95 \% \mathrm{CI}[0.006,0.076]$, but not preference for nonbeating, $a_{2} b_{2}=-0.001, S E=0.008,95 \% \mathrm{CI}[-0.017,0.016]$. The total indirect effect for the mediators reached significance, $f=0.037, S E=0.020,95 \%$ CI $[0.001,0.080]$. The total effect of age was significant, $c=0.076, S E=0.028,95 \% \mathrm{CI}[0.019,0.131]$, but after accounting for the mediators, the direct effect of age was reduced to non-significance, $c^{\prime}=0.039, S E=0.027,95 \%$ CI $[-0.013,0.092]$. Together these results suggest that age relates to preference for consonant intervals because of a co-occurring increase in preference for harmonicity, as shown in Figure 5.

While age-related increases in preference for harmonicity explained the age-related increase in preference for consonance, it could be that factors other than age contribute to the effects of harmonicity. A series of correlations (Holm-Bonferroni corrected) revealed a significant positive relationship between preference for harmonicity and preference for consonance within each age group $($ Age $6: r(21)=.59, p=.010$; Age 8: $r(23)=.48, p=.029$; Age 10: $r(21)=.69, p=.001$; Adult: $r(24)=.47, p=.029)$. This result suggests that a simple marker of experience such as age does not fully account for the harmonicity-consonance relationship in childhood; other experiences or pre-existing differences contribute as well. Moreover, this result shows that even in young children, harmonicity preferences contribute substantially to individual differences in the preference for consonance. In contrast, preference for non-beating stimuli did not correlate with preference for consonance within any age group, $p$ s $>.2$ (uncorrected).

\section{Discussion}


Preferences for consonant intervals increased from 6 years of age to adulthood, and by 10 years of age were statistically similar to those of adults. The age-related increase in preference for consonance occurred in the context of an overall age-related decline in ratings. Essentially, the decline was more pronounced for dissonant than for consonant intervals. Among dissonant intervals, the smaller intervals (i.e., minor and major second) showed the greatest reduction in ratings. A simple age-related improvement in discernment cannot account for these results. Ratings declined with age on the control task (i.e., affective vocalizations), as for the consonant/dissonant stimuli, reflecting a tendency toward a more balanced or centered use of the rating scale, but there was no age-related enhancement in the preference for happy vocalizations.

These age-related increases in consonance preferences are generally consistent with previous findings in children (Valentine, 1913), with the effects of musical training on Western adults' preferences (McDermott et al., 2010), and with cross-cultural differences in preferences presumed to be driven by differences in exposure to Western music (McDermott et al., 2016). The significant preferences for consonance in 6-year-olds are at odds with the absence of consonance preferences in the 6- and 7-year-olds tested by Valentine (1913). Increases in exposure to music over the past century, stemming from dramatic increases in the ease of access to music, may account for the earlier emergence of consonance preferences in contemporary children. Despite the importance of documenting the age of emergence of consonance preferences, there are numerous barriers to doing so, in particular the difficulty of obtaining reliable evaluative judgments from younger children.

Developmental increases in the preference for consonance were fully mediated by the preference for harmonicity rather than the aversion to beating. This finding is in line with previous findings that only harmonicity predicted individual differences in adults' preference for 
consonance (McDermott et al., 2010). Although there was a significant effect of age on preferences for harmonicity and for consonance, these relations were less robust than the relation between harmonicity and consonance preferences. In other words, age accounted for a very small proportion of the variance in preferences for consonance.

One explanation for the relatively modest age-related change in preferences is the imperfect correspondence of age to cumulative musical exposure. The participants had no formal training or very limited training, but it is likely that most of them participated in musical activities of some kind (e.g., singing, dancing) to a greater or lesser extent. Information about casual musical exposure, while potentially useful, is difficult to access and quantify, in contrast to information about formal exposure or training. The consequences of formal training on children's and adults' aesthetic preferences for intervals were explored in Study 2.

\section{Study 2}

Study 1 showed age-related changes in preferences for consonance in nonmusician children and adults, consistent with the idea that incidental exposure to music has notable consequences on aesthetic evaluations of simultaneous tone combinations. The role of formal musical training was examined in children and adults in Study 2. Recruitment of "child musicians" was restricted to 10-year-olds to ensure sufficient numbers of children with the requisite musical training.

\section{Method}

Participants. Musician participants were approximately $10(n=18,10$ female, $M=10.2$, $S D=0.2$, range $=9.9-10.5$ years $)$ and 21 years of age $(n=22,13$ female, $M=20.6, S D=3.8$, range $=18.1-32.5$ years $).$ Sample sizes were determined using effect sizes and variability reported in previous research with participants who varied in musical experience (McDermott et 
al, 2016). All participants were born in Canada. Child musicians had 2 or more years of lessons $(M=4.2, S D=1.9$, range $=2.0-8.2$ years $)$, with lessons still in progress, and adult musicians had 5 or more years of lessons $(M=8.2, S D=3.0$, range $=5.0-14.0$ years $)$. Three additional children were excluded because of perseverative responding on one or more stimulus sets, according to criteria noted in Study $1(n=2)$, or illness on the day of testing $(n=1)$. Comparison data for nonmusicians were taken from the 10-year-olds and young adults in Study 1. Within each age group, musicians and nonmusicians did not differ in age, $p \mathrm{~s}>.1$. This research was approved by the Human Research Ethics Program of the University of Toronto.

Stimuli, apparatus, and procedure. All aspects of design and presentation were identical to Study 1.

\section{Results}

Musicianship and use of rating scale. Ratings in the vocalization set were averaged over trials with positive vocalizations ( $n=5$ happy trials) and negative vocalizations ( $n=10 \mathrm{sad}$ or fearful trials). Descriptive statistics are shown in Figure 6A. A mixed-model ANOVA compared mean ratings, with vocalization type (positive, negative) as a within-participant factor and age group (10, adult) and musicianship (musician, nonmusician) as between-participants factors. As expected, there was a significant main effect for vocalization type, $F(1,85)=172.07$, $p<.001, \eta_{p}{ }^{2}=0.67$, indicating higher ratings for positive vocalizations $(M=2.98, S D=0.61)$ than negative vocalizations $(M=1.75, S D=0.62)$. There was also a significant main effect of age, $F(1,85)=5.93, p=.017, \eta_{p}{ }^{2}=0.07$, with adults providing lower ratings $(M=2.27, S D=$ $0.34)$ than children $(M=2.48, S D=0.49)$ regardless of stimulus favorability. However, there were no other main effects or interactions, $p \mathrm{~s}>.1$, which suggests that the rating scale was used to differentiate liked from disliked stimuli in a similar manner across age and musicianship. 
Musicianship and preference for consonance. As in Study 1, ratings within two stimulus sets (synthetic, sung) were averaged according to consonance or dissonance (see Table 1), yielding four scores per participant (see Figures 6B-C). Figure 7 displays descriptive statistics for ratings by individual intervals across stimulus sets, age groups, and musicianship. Figure 8A summarizes preference for consonance across ages and musicianship in Studies 1 and 2.

An initial mixed-model ANOVA examined whether ratings were affected by withinparticipant factors of consonance (consonant, dissonant) and stimulus set (synthetic, sung), and between-participant factors of age group (10, adult) and musicianship (musician, nonmusician). There was no main effect for musicianship, $p>.8$, but there was a significant main effect for consonance, $F(1,85)=175.28, p<.001, \eta_{p}{ }^{2}=.67$, and an interaction between musicianship and consonance, $F(1,85)=16.01, p<.001, \eta_{p}{ }^{2}=.16$, which was driven by a larger preference for consonance (i.e., ratings for consonant minus dissonant trials) in musicians $(M=0.79, S D=$ $0.51)$ compared to nonmusicians $(M=0.41, S D=0.36)$. There was a main effect of stimulus set, $F(1,85)=17.36, p<.001, \eta_{p}{ }^{2}=.17$, which was qualified by a two-way interaction between stimulus set and age group, $F(1,85)=4.99, p=.028, \eta_{p}^{2}=.05$. Overall ratings in the sung stimulus set did not differ between 10 -year-olds $(M=2.63, S D=0.46)$ and adults $(M=2.62, S D$ $=0.33), t(87)=0.18, p>.8$, but overall ratings in the synthetic stimulus set were higher for 10 year-olds $(M=2.55, S D=0.55)$ than adults $(M=2.33, S D=0.27), t(87)=2.40, p=.018$. There was also a two-way interaction between stimulus set and consonance, $F(1,85)=4.79, p=.031$, $\eta_{p}{ }^{2}=.05$, which indicated that across all participants there was a greater preference for consonance in the sung stimulus set $(M=0.62, S D=0.54)$ than in the synthetic stimulus set $(M=$ $0.54, S D=0.46), t(88)=2.16, p=.033$. Finally, there was an interaction between age and 
consonance, $F(1,85)=7.92, p=.006, \eta_{p}{ }^{2}=.09$, which emerged due to a greater preference for consonance in adults $(M=0.69, S D=0.47)$ than 10 -year-olds $(M=0.45, S D=0.44)$. There were no other effects or higher-order interactions in the primary ANOVA, $p \mathrm{~s}>.05$. In sum, experience, whether by training or age, enhanced the preference for consonance.

In two follow-up independent-samples $t$-tests, we asked whether musicianship affected ratings for consonant trials only, or dissonant trials only (i.e., collapsed across stimulus set). Neither comparison was significant after Holm-Bonferroni correction (corrected $p$-values displayed), but musicians, collapsed across age group, gave higher ratings on consonant trials ( $M$ $=2.91, S D=0.38)$ than nonmusicians $(M=2.74, S D=0.35)$ in absolute terms, $t(87)=2.24, p=$ .055 , and their ratings on dissonant trials $(M=2.13, S D=0.49)$ were lower than those of nonmusicians $(M=2.33, S D=0.45)$ in absolute terms, $t(87)=1.99, p=.055$.

Musicianship and aversion to beating or inharmonicity. To assess listeners' responses to beating, 2 scores were calculated by averaging ratings for trials of tone pairs according to presentation type (diotic, dichotic), with 12 trials per score (i.e., repeating the analysis of Study 1). Results are shown in Figure 6D. A mixed-model ANOVA comparing ratings, with presentation type as a repeated factor and age group (10, adult) and musicianship (musician, nonmusician) as between-participants factors, revealed significant main effects of presentation type, $F(1,85)=141.05, p<.001, \eta_{p}{ }^{2}=.62$, and age group, $F(1,85)=7.77, p=.007, \eta_{p}^{2}=.08$. There was a significant interaction between age group and presentation type, $F(1,85)=4.79, p=$ $.031, \eta_{p}{ }^{2}=.05$, in line with the developmental pattern observed in Study 1 (i.e., greater preference for non-beating stimuli in adults, see Figure 8B). However, there were no main effects or interactions involving musicianship, $p s>.1$ (see Figure 6D), consistent with the results 
of McDermott et al. (2010), who found no correlation between aversions to beating and musical experience.

As in Study 1 and following McDermott et al. (2016), harmonicity ratings were assessed by calculating two scores per participant, one combining eight harmonic trials and four pure-tone trials (hereafter harmonic score) and another combining eight inharmonic trials and four lowdichotic tone-pair trials (hereafter inharmonic score). Results are shown in Figure 6E. A mixedmodel ANOVA comparing ratings, with harmonicity as a repeated factor and age group (10, adult) and musicianship (musician, nonmusician) as between-participants factors, revealed a significant main effect of harmonicity, $F(1,85)=117.18, p<.001, \eta_{p}{ }^{2}=.58$, no main effects of age group or musicianship, $F \mathbf{s}<1$, and an interaction between musicianship and harmonicity, $F(1,85)=8.87, p=.004, \eta_{p}{ }^{2}=.10$. The difference between ratings for harmonic and inharmonic stimuli (see Figure 6E) was larger for musicians $(M=0.93, S D=0.73)$ than for nonmusicians $(M$ $=0.52, S D=0.53)$, consistent with the results of McDermott et al. (2010), who found strong correlations between preferences for harmonic stimuli and musical experience. There was no interaction between age group and harmonicity, $F(1,85)=2.84, p=.096$, and no three-way interaction, $F(1,85)=2.72, p=.103$, but given the trends in Figure $8 \mathrm{C}$, the absence of a threeway interaction is tentative. In any case, were the preference for harmonicity to interact with age group and musicianship in future studies with more power, it would plausibly be driven by the unavoidable difference in years of formal training between 10 -year-old musicians and adult musicians (see Participants).

Figure 8 summarizes the results of both studies, illustrating that while all three preferences (consonance, beating, and harmonicity) increased with age, only consonance and harmonicity preferences increased with musicianship. The effect of musicianship on preference 
for harmonicity raised the possibility that harmonicity mediated the relationship between musical training and preference for consonance. When considered as a continuous variable across the sample (i.e., collapsing across age group), years of musical training correlated positively with both preference for consonance, $r(87)=.42, p<.001$, and preference for harmonicity, $r(87)=$ $.52, p<.001$. (Preference for non-beating was excluded from consideration as a mediator because it was not related to musical training, $p>$.7). A mediation analysis using the bootstrap approach (Preacher \& Hayes, 2008; 5,000 resamples) revealed that years of musical training affected preference for consonance as a function of its relationship with preference for harmonicity, $a b=0.036, S E=0.010,95 \% \mathrm{CI}[0.016,0.056]$. The total effect of musical training was significant, $c=0.067, S E=0.013,95 \%$ CI [0.041, 0.092], and after accounting for the indirect effect of the mediator, the direct effect of musical training was significant but reduced, $c^{\prime}=0.031, S E=0.008,95 \%$ CI $[0.015,0.049]$. These results, shown in Figure 9, suggest that increases in preference for harmonicity partially account for the relationship between musical training and preference for consonant intervals. In turn, the harmonicity-consonance relationship did not depend on years of musical training: partial correlations (Holm-Bonferroni corrected) between preference for harmonicity and preference for consonance, controlling for years of musical training, revealed strong relationships across the sample, $r(86)=.72, p<.001$, and within all four age/musicianship subgroups (10-year-old nonmusicians, $r(20)=.67, p=.001 ; 10$ year-old musicians, $r(15)=.78, p<.001$; adult nonmusicians, $r(23)=.50, p=.011$; adult musicians, $r(19)=.84, p<.001)$.

\section{Discussion}

Consonance preferences were affected by age and formal musical training. Across both stimulus sets, musical training enhanced the preference for consonance additively with effects of 
age. Similarly, Study 2 replicated the age-related increase in preference for consonance observed in Study 1, despite a more limited age range. The effects of musicianship, when present, involved enhanced preferences, in line with previous research (Dellacherie et al. 2011; McDermott et al., 2010, 2016). Preference for consonance may be similar in 10-year-olds and adult nonmusicians (Study 1), but it is amenable to enhancement from musical training.

Sung intervals elicited a greater preference for consonance than did synthetic materials. Sung and synthetic materials were treated differently by the age groups in Study 1 when taking overall ratings into account (see the absence of an overall rating decline with age for sung materials in Figure 3C), but this effect did not interact with preference for consonance, and roughly half of those participants were included in the analysis in Study 2. One possibility involves differential processing of sung and instrumental materials (Weiss et al., 2016), which enhanced the preferences of nonmusician 10-year-olds. The preference for sung over synthetic materials and a greater preference for consonance in sung materials is consistent with the prevalence of vocal music across cultures (Savage et al., 2015). Rapid fluctuations in fundamental frequency (e.g., jitter, vibrato), which are inherent in vocal tones but not in most instrumental tones, may also affect listeners' preferences.

Musicianship did not influence ratings for beating or non-beating stimuli, but it affected ratings for harmonic or inharmonic stimuli, as in previous research (McDermott et al., 2010). Together with the effect of age on harmonicity in Study 1, the current effect of training on harmonicity supports the idea that musical experience shapes harmonicity preferences. Moreover, the preference for harmonicity partially mediated the relationship between musical training and preference for consonance, reinforcing the role of experience in preferences for consonance. The effect of musicianship was not fully mediated, so its role in preferences for 
harmonic and interval stimuli remains unclear. Regardless, the partial mediation provides evidence that changes in responses to acoustic features contribute to changes in preference for consonance. Harmonicity correlated strongly with preferences for consonance in musicians even after controlling for years of musical training. Other differences in experience or pre-existing preferences must account for the remainder of the variance in the harmonicity-consonance relationship.

In principle, musically trained participants may have pursued lessons because of stronger pre-existing preferences for consonance or harmonicity. The cross-sectional, quasi-random selection of participants in the current design precludes causal inference, but future research with longitudinal design and random assignment to training condition could address this concern. However, biases for consonance or harmonicity that exist prior to training would be unlikely to explain the link between years of musical training and acoustic preferences for participants of similar age (e.g., McDermott et al., 2010) or differences in consonance ratings between participants with degrees in music and those with substantial musical training but no degree (Arthurs, Beeston, \& Timmers, 2017). Among adult musicians in Study 2, for example, there was a significant positive relationship between overall preference for consonance and years of musical training, $r(20)=.48, p=.024$. A similar relationship between preference for harmonic stimuli and years of musical training did not reach significance, $r(20)=.40, p=.068$, perhaps for lack of power.

\section{General Discussion}

The present research examined children's and adults' aesthetic evaluations of consonant and dissonant simultaneous intervals. Adults and children 6-10 years of age with limited musical training (Study 1) and 10-year-old children and adults with musical training (Study 2) exhibited a 
preference for consonant intervals, but the magnitude of the preference changed as a function of age and musicianship (see Figure 8). The mean difference in ratings between consonant and dissonant intervals in adult musicians was 2.1 times as large as that for adult nonmusicians, which in turn was 1.9 times as large as that for the youngest children (6-year-olds). Moreover, by age 10 , nonmusician children did not differ statistically from nonmusician adults in their preference for consonance. By contrast, preferences for positive affective vocalizations (e.g., laughs versus gasps) did not differ across these groups. We conclude that experience plays a critical role in the development of consonance preferences, in line with the effects of musical experience on adults' preferences (Guernsey, 1928; McDermott et al., 2010; McLachlan et al., 2013; McDermott et al., 2016).

The effect of age on consonance preferences in Study 1 and the effect of years of musical training on consonance preferences in Study 2 were mediated in full (Study 1) or in part (Study 2) by the preference for harmonic stimuli, whereas aversion to beating failed to mediate either effect. The results thus contribute to the accumulating evidence that enculturation influences preferences for consonance and harmonicity by documenting effects of age and musical training in children. Native Amazonians who are isolated from Western music (the Tsimane') are averse to beating but show no preference for harmonicity or for intervals that conform to Western notions of consonance (McDermott et al., 2016). Listeners with impaired pitch perception whose experience of Western tonality and harmony is limited by biology rather than exposure show a pattern of preferences like those of the Tsimane' (Cousineau et al., 2012). Among typically developing North American participants, years of music lessons correlate positively with preferences for harmonicity and for consonance but not with aversion to beating (McDermott et al., 2010). These studies are inconsistent with the classical account of consonance preferences as 
driven by the absence of beating (Helmholtz, 1895; Hutchinson \& Knopoff, 1978; Sethares, 2005). Instead, the aversion to beating may represent an aesthetic evaluation that is orthogonal to the preference for consonance, perhaps because beating is modest in naturally occurring musical intervals and varies considerably with instrumental timbre. Note, however, that the current results do not dismiss the potential importance of beating in the history of Western music. Contemporary preferences may differ from those of previous generations because of differences in exposure to dissonance in music.

The effects of age and musical training on preferences for harmonic and consonant stimuli with age are incompatible with a fixed, innate preference for harmonic sounds or tonal fusion (Dewitt \& Crowder, 1987). According to the cognitive incongruence model of dissonance (McLachlan et al., 2013), negative affect is experienced when there is incongruence between intervals and priming from learned chord templates in long-term memory. However, children's higher ratings in Study 1 present a challenge for theories of aesthetic preference based on processing fluency (Reber, Schwarz, \& Winkielman, 2004). Child participants in the current study rated dissonant intervals more favorably than adult participants, a finding reported by Valentine (1913) more than a century ago. This finding cannot be attributed to greater experience with dissonant intervals. Instead, children may experience less displeasure from dissonant intervals because of a relatively flexible template of consonance. This flexible template may result from limited exposure to music in general or from qualitative differences in the processing of musical stimuli, and may relate to developmental differences in other aspects of implicit knowledge for music such as key (Corrigall \& Trainor, 2010; Corrigall \& Trainor, 2014; Kragness \& Trainor, 2018), harmony (Folland et al., 2015; He \& Trainor, 2009), and rhythm (Hannon \& Trehub, 2005a, b; Soley \& Hannon, 2010). Using the current findings as a baseline, 
future research could manipulate short-term exposure to dissonant chords in children and adults to probe the degree to which mental chord templates, and in turn behavioral responses, are amenable to change at different ages. For example, exposure to consonant or dissonant patterns immediately prior to testing biases infants' subsequent listening in favor of the familiarized stimulus (Plantinga \& Trehub, 2014). Another approach would require children or adults to "tune" the higher tone in an interval to a preferred pitch (e.g., Hutchins \& Peretz, 2012), restricting the range of the tuner to facilitate comparisons of specific intervals.

There is a long and complex web of explanations about the mathematical, acoustic, biological, or experiential bases of listeners' aesthetic preference for consonance (e.g., Bidelman, 2013; Ebeling, 2008; Fishman, et al., 2001; Helmholtz, 1895; Kameoka \& Kuriyagawa, 1969; McDermott et al., 2010; Plomp \& Levelt, 1965; Stumpf, 1890; Terhardt, 1974; Tramo et al., 2001). By relying primarily on listeners with extensive exposure to Western music (e.g., 'WEIRD' subjects; Henrich, Heine, \& Norenzayan, 2012) or using obtuse descriptors (e.g., “clearness" and "turbidity"; Kameoka \& Kuriyagawa, 1969), researchers generate models of consonance that may fail to predict aesthetic preferences in children or in non-Western listeners. Our findings and those of Valentine (1913), together with cross-cultural evidence of indifference to dissonance (McDermott et al., 2016), historical changes in conceptions of consonance (Tenney, 1988), effects of musical experience on consonance preferences in Western adults (McDermott et al., 2010), and the malleability of infants" "preferences" for consonance (Plantinga \& Trehub, 2014) should insulate us against notions of innate or fixed aesthetic preferences for consonance. 


\section{Context of the Research}

The ideas for this study originated from discussions among the authors, who lamented the absence of modern research on age-related changes in consonance preferences. At the same time, the authors were intrigued by the finding that adult listeners with little apparent exposure to Western music do not exhibit consonance preferences (McDermott et al., 2016). Because such preferences are considered to be a fundamental aspect of music cognition, it seemed important to establish their developmental timetable, especially the emergence of adultlike preferences for consonance. The results raise several natural follow-up questions. What is the relationship between passive exposure to music across development and consonance preferences? Would the developmental pattern of preferences differ for more ecologically valid musical stimuli? Are aesthetic preferences for consonance absent in preschool children, who exhibit excellent discrimination skills but limited implicit knowledge of Western musical conventions? We hope the current results will spur research on these questions and others. 


\section{Acknowledgements}

We thank Chella Velkannan, Zuzanna Jurewicz, Leila Baisyrymova, and Balpreet Dhugga for assistance in data collection. 


\section{References}

Alain, C., Arnott, S. R., \& Picton, T. W. (2001). Bottom-up and top-down influences on auditory scene analysis: Evidence from event-related brain potentials. Journal of Experimental Psychology: Human Perception and Performance, 27, 1072-1089.

\section{https://doi.org/10.1037//0096-1523.27.5.1072}

Arthurs, Y., Beeston, A. V., \& Timmers, R. (2017). Perception of isolated chords: Examining frequency of occurrence, instrumental timbre, acoustic descriptors, and musical training. Psychology of Music, 46, 662-681. doi: https://doi.org/10.1177/0305735617720834

Belin, P., Fillion-Bilodeau, S., \& Gosselin, F. (2008). The Montreal Affective Voices: A validated set of nonverbal affect bursts for research on auditory affective processing. Behavior Research Methods, 40, 531-539. https://doi.org/10.3758/BRM.40.2.531

Bidelman, G. M. (2013). The role of the auditory brainstem in processing musically relevant pitch. Frontiers in Psychology, 4, Article 264. https://doi.org/10.3389/fpsyg.2013.00264

Bregman, A. S. (1990). Auditory scene analysis. Cambridge, MA: MIT Press.

Brown, S., \& Jordania, J. (2013). Universals in the world's musics. Psychology of Music, 41, 229-248. http://doi.org/10.1177/0305735611425896

Cazden, N. (1980). The definition of consonance and dissonance. International Review of the Aesthetics and Sociology of Music, 11, 123-168. 
Corrigall, K. A., \& Trainor, L. J. (2014). Enculturation to musical pitch structure in young children: Evidence from behavioral and electrophysiological methods. Developmental Science, 17, 142-158. https://doi.org/10.1111/desc.12100

Corrigall, K., \& Trainor, L. (2010). Musical enculturation in preschool children: Acquisition of key and harmonic knowledge. Music Perception, 28, 195-200. https://doi.org/10.1525/MP.2010.28.2.195

Cousineau, M., McDermott, J. H., \& Peretz, I. (2012). The basis of musical consonance as revealed by congenital amusia. Proceedings of the National Academy of Sciences, 109, 19858-19863. https://doi.org/10.1073/pnas.1207989109

Dellacherie, D., Roy, M., Hugueville, M., Peretz, I., \& Samson, S. (2011). The effect of musical experience on emotional self-reports and psychophysiological responses to dissonance. Psychophysiology, 48, 337-349. https://doi.org/10.1111/j.1469-8986.2010.01075.x

Dewitt, L. A., \& Crowder, R. G. (1987). Tonal fusion of consonant musical intervals: The oomph in Stumpf. Perception \& Psychophysics, 41, 73-84. https://doi.org/10.3758/BF03208216

Draganova, R., Eswaran, H., Murphy, P., Huotilainen, M., Lowery, C., \& Preissl, H. (2005). Sound frequency change detection in fetuses and newborns, a magnetoencephalographic study. NeuroImage, 28, 354-361. https://doi.org/10.1016/j.neuroimage.2005.06.011 
Ebeling, M. (2008). Neuronal periodicity detection as a basis for the perception of consonance: A mathematical model of tonal fusion. Journal of the Acoustical. Society of America, 124, 2320-2329. doi:10.1121/1.2968688

Fishman, Y.I., Volkov, I.O., Noh, M.D., Garell, P.C., Bakken, H., Arezzo, J.C., Howard, M.A., \& Steinschneider, M. (2001). Consonance and dissonance of musical chords: Neural correlates in auditory cortex of monkeys and humans. Journal of Neurophysiology, 86, 2761-2788. doi: $\underline{10.1152 / j n .2001 .86 .6 .2761}$

Folland, N. A., Butler, B. E., Payne, J. E., \& Trainor, L. J. (2015). Cortical representations sensitive to the number of perceived auditory objects emerge between 2 and 4 months of age: Electrophysiological evidence. Journal of Cognitive Neuroscience, 27, 1060-1067. https://doi.org/10.1162/jocn_a_00764

Folland, N. A., Butler, B. E., Smith, N. A., \& Trainor, L. J. (2012). Processing simultaneous auditory objects: Infants' ability to detect mistuning in harmonic complexes. Journal of the Acoustical Society of America, 131, 993-997. https://doi.org/10.1121/1.3651254

Guernsey, M. (1928). The role of consonance and dissonance in music. American Journal of Psychology, 40, 173-204.

Háden, G. P., Stefanics, G., Vestergaard, M. D., Denham, S. L., Sziller, I., \& Winkler, I. (2009). Timbre-independent extraction of pitch in newborn infants. Psychophysiology, 46, 69-74. https://doi.org/10.1111/j.1469-8986.2008.00749.x 
Hannon, E. E., \& Trehub, S. E. (2005). Metrical categories in infancy and adulthood.

Psychological Science, 16, 48-55. https://doi.org/10.1111/j.0956-7976.2005.00779.x

Hannon, E. E., \& Trehub, S. E. (2005). Tuning in to musical rhythms: Infants learn more readily than adults. Proceedings of the National Academy of Sciences of the United States of America, 102, 12639-12643. https://doi.org/10.1073/pnas.0504254102

He, C., \& Trainor, L. J. (2009). Finding the pitch of the missing fundamental in infants. Journal of Neuroscience, 29, 7718-8822. https://doi.org/10.1523/JNEUROSCI.0157-09.2009

Helmholtz, H. L. F. (1895). On the sensations of tone (3rd ed.). New York, NY: Longmans, Green, and Co.

Henrich, J., Heine, S. J., \& Norenzayan, A. (2010). The weirdest people in the world? Behavioral and Brain Sciences, 33, 61-83. https://doi.org/10.1017/S0140525X0999152X

Houston-Price, C., \& Nakai, S. (2004). Distinguishing novelty and familiarity effects in infant preference procedures. Infant and Child Development, 13, 341-348. https://doi.org/10.1002/icd.364

Hutchins, S. M., \& Peretz, I. (2012). A frog in your throat or in your ear? Searching for the causes of poor singing. Journal of Experimental Psychology: General, 141, 76-97. https://doi.org/10.1037/a0025064

Hutchinson, W., \& Knopoff, L. (1978). The acoustic component of Western consonance. Journal of New Music Research, 7, 1-29. https://doi.org/10.1080/09298217808570246 
Jacoby, N., \& McDermott, J. H. (2017). Integer Ratio Priors on Musical Rhythm Revealed Cross-culturally by Iterated Reproduction. Current Biology, 27, 359-370. https://doi.org/10.1016/j.cub.2016.12.031

Jordania, J. (2006). Who asked the first question? The origins of human choral singing, intelligence, language and speech. Tblisi, Russia: Logos.

Kameoka, A., \& Kuriyagawa, M. (1969). Consonance Theory Part I: Consonance of Dyads. Journal of the Acoustical Society of America, 45, 1451-1459. https://doi.org/10.1121/1.1911623

Kragness, H. E., \& Trainor, L. J. (2018). Young children pause on phrase boundaries in selfpaced music listening: The role of harmonic cues. Developmental Psychology, 54, 842-856. https://doi.org/10.1037/dev0000405

Masataka, N. (2006). Preference for consonance over dissonance by hearing newborns of deaf parents and of hearing parents. Developmental Science, 9, 46-50. https://doi.org/10.1111/j.1467-7687.2005.00462.x

McDermott, J. H., Lehr, A. J., \& Oxenham, A. J. (2010). Individual differences reveal the basis of consonance. Current Biology, 20, 1035-1041. https://doi.org/10.1016/j.cub.2010.04.019

McDermott, J. H., Schultz, A. F., Undurraga, E. A., \& Godoy, R. A. (2016). Indifference to dissonance in native Amazonians reveals cultural variation in music perception. Nature, 25, 21-25. https://doi.org/10.1038/nature18635 
McLachlan, N., Marco, D., Light, M., \& Wilson, S. (2013). Consonance and pitch. Journal of Experimental Psychology: General, 142, 1142-1158. https://doi.org/10.1037/a0030830

McPherson, M. J., \& McDermott, J. H. (2018). Diversity in pitch perception revealed by task dependence. Nature Human Behaviour, 2, 52-66. https://doi.org/10.1038/s41562-017-02618

Norman-Haignere, S., \& McDermott, J. H. (2016). Distortion products in auditory fMRI research: measurements and solutions. NeuroImage, 129, 401-413. https://doi.org/10.1016/j.neuroimage.2016.01.050

Novitski, N., Huotilainen, M., Tervaniemi, M., Näätänen, R., \& Fellman, V. (2007). Neonatal frequency discrimination in 250-4000-Hz range: Electrophysiological evidence. Clinical Neurophysiology, 118, 412-419. https://doi.org/10.1016/j.clinph.2006.10.008

Olsho, L. W., Koch, E. G., Carter, E. A., Halpin, C. F., \& Spetner, N. B. (1988). Pure-tone sensitivity of human infants. Journal of the Acoustical Society of America, 84, 1316-1324. https://doi.org/10.1121/1.396630

Oxenham, A. J. (2013). The perception of musical tones. In D. Deutsch (Ed.), The Psychology of Music (3rd ed., pp. 1-33). San Diego, CA: Academic Press.

Parncutt, R., \& Hair, G. (2011). Consonance and dissonance in music theory and psychology: Disentangling dissonant dichotomies. Journal of Interdisciplinary Music Studies, 5, 119166. https://doi.org/10.4407/jims.2011.11.002 
Plantinga, J., \& Trehub, S. E. (2014). Revisiting the innate preference for consonance. Journal of Experimental Psychology: Human Perception and Performance, 40, 40-49. https://doi.org/10.1037/a0033471

Plomp, R., \& Levelt, W. J. M. (1965). Tonal consonance and critical bandwidth. Journal of the Acoustical Society of America, 38, 548-560.

https://doi.org/http://dx.doi.org/10.1121/1.1909741

Popham, S., Boebinger, D., Ellis, D. P. W., Kawahara, H., \& McDermott, J. H. (2018).

Inharmonic speech reveals the role of harmonicity in the cocktail party problem. Nature Communications, 9, 2122. https://doi.org/10.1038/s41467-018-04551-8

Preacher, K. J., \& Hayes, A. F. (2008). Asymptotic and resampling strategies for assessing and comparing indirect effects in multiple mediator models. Behavior Research Methods, 40, 879-891. https://doi.org/10.3758/BRM.40.3.879

Pressnitzer, D., \& Patterson, R. D. (2001). Distortion products and the perceived pitch of harmonic complex tones. In D. J. Breebart, A. J. M. Houtsma, A. Kohlrausch, V. F. Prijs, \& R. Schoonoven (Eds.), Physiological and Psychophysical Bases of Auditory Function (pp. 97-104). Maastricht, The Netherlands: Shaker Publishing.

Purves, D. (2017). Music as biology. Cambridge, MA: Harvard University Press.

Reber, R., Schwarz, N., \& Winkielman, P. (2004). Processing fluency and aesthetic pleasure: Is beauty in the perceiver's processing experience? Personality and Social Psychology Review, 8, 364-382. https://doi.org/10.1207/s15327957pspr0804_3 
Rutschmann, J., \& Rubinstein, L. (1965). Binaural Beats and Binaural Amplitude-Modulated Tones: Successive Comparison of Loudness Fluctuations. Journal of the Acoustical Society of America, 38, 759-768. https://doi.org/10.1121/1.1909802

Savage, P. E., Brown, S., Sakai, E., \& Currie, T. E. (2015). Statistical universals reveal the structures and functions of human music. Proceedings of the National Academy of Sciences, 112, 8987-8992. http://doi.org/10.1073/pnas.1414495112

Schellenberg, E. G., \& Trehub, S. E. (1996). Natural musical intervals: Evidence from infant listeners. Psychological Science, 7, 272-277. Retrieved from https://www.jstor.org/stable/40062961

Schneider, B. A., Avivi-Reich, M., \& Mozuraitis, M. (2015). A cautionary note on the use of the Analysis of Covariance (ANCOVA) in classification designs with and without withinsubject factors. Frontiers in Psychology, 6, 1-12. https://doi.org/10.3389/fpsyg.2015.00474

Sethares, W. A. (2005). Tuning, timbre, spectrum, scale. London, UK: Springer Science \& Business Media.

Soley, G., \& Hannon, E. E. (2010). Infants prefer the musical meter of their own culture: A cross-cultural comparison. Developmental Psychology, 46, 286-292. https://doi.org/10.1037/a0017555

Stumpf, C. (1890). Tonpsychologie. Leipzig, Germany: Verlag S. Hirzel.

Tenney, J. (1988). A history of “consonance” and “dissonance.” New York. New York, NY: Excelsior. 
Tenzer, M. (1991). An introduction to Balinese music. Seattle, WA: Periplus Editions.

Terhardt, E. (1974). On the perception of periodic sound fluctuations (roughness). Acta Acustica united with Acustica, 30, 201-213.

Terhardt, E. (1974). Pitch, consonance, and harmony. Journal of the Acoustical Society of America, 55, 1061-1069. http://dx.doi.org/10.1121/1.1914648

Trainor, L. J., \& Heinmiller, B. M. (1998). The development of evaluative responses to music: Infants prefer to listen to consonance over dissonance. Infant Behavior and Development, 21, 77-88. https://doi.org/10.1016/S0163-6383(98)90055-8

Trainor, L. J., Tsang, C. D., \& Cheung, V. H. W. (2002). Preference for sensory consonance in 2- and 4-month-old infants. Music Perception, 20, 187-194. https://doi.org/10.1525/mp.2002.20.2.187

Tramo, M. J., Cariani, P. A., \& Delgutte, B. (2001). Neurobiological foundations for the theory of harmony in Western tonal music. Annals of the New York Academy of Sciences, 930, 92116. https://doi.org/10.1111/j.1749-6632.2001.tb05727.x

Tramo, M. J., Cariani, P. A., Delgutte, B., \& Braida, L. D. (2006). Neurobiological foundations for the theory of harmony in Western tonal music. Annals of the New York Academy of Sciences, 930, 92-116. https://doi.org/10.1111/j.1749-6632.2001.tb05727.x

Valentine, C. W. (1913). The aesthetic appreciation of musical intervals among school children and adults. British Journal of Psychology, 6, 190-216. https://doi.org/10.1017/CBO9781107415324.004 
Vassilakis, P. N. (2005). Auditory roughness as means of musical expression. Selected Reports in Ethnomusicology, 12, 119-144.

Vetter, R. (1989). A retrospect on a century of gamelan tone measurements. Ethnomusicology, 33, 217-227. https://doi.org/10.2307/924396

Weisleder, A., \& Fernald, A. (2013). Talking to children matters: Early language experience strengthens processing and builds vocabulary. Psychological Science, 24, 2143-2152. https://doi.org/10.1177/0956797613488145

Weiss, M. W., Trehub, S. E., Schellenberg, E. G., \& Habashi, P. (2016). Pupils dilate for vocal or familiar music. Journal of Experimental Psychology: Human Perception and Performance, 42, 1061-1065. https://doi.org/10.1037/xhp0000226

Zentner, M. R., \& Kagan, J. (1998). Infants' perception of consonance and dissonance in music. Infant Behavior and Development, 21, 483-492. https://doi.org/10.1016/S0163$\underline{6383(98) 90021-2}$ 


\begin{tabular}{llll} 
Interval & Semitones & Type & Stimulus sets \\
\hline unison & 0 & - & Synthetic, Sung \\
min2 & 1 & D & Synthetic, Sung \\
maj2 & 2 & D & Synthetic, Sung \\
min3 & 3 & C & Synthetic, Sung \\
maj3 & 4 & C & Synthetic, Sung \\
perf4 & 5 & C & Synthetic, Sung \\
tritone & 6 & D & Synthetic, Sung \\
perf5 & 7 & C & Synthetic, Sung \\
min6 & 8 & C & Synthetic, Sung \\
maj6 & 9 & C & Synthetic, Sung \\
min7 & 10 & D & Synthetic, Sung \\
maj7 & 11 & D & Synthetic, Sung \\
octave & 12 & C & Synthetic
\end{tabular}

Table 1. List of intervals and their classification as consonant (C) or dissonant (D) as in previous research (McDermott et al., 2010). Because of differences in stimulus design (see Stimuli), the synthetic intervals set included intervals from unison to octave, and the sung set included intervals from unison to maj7. 
A

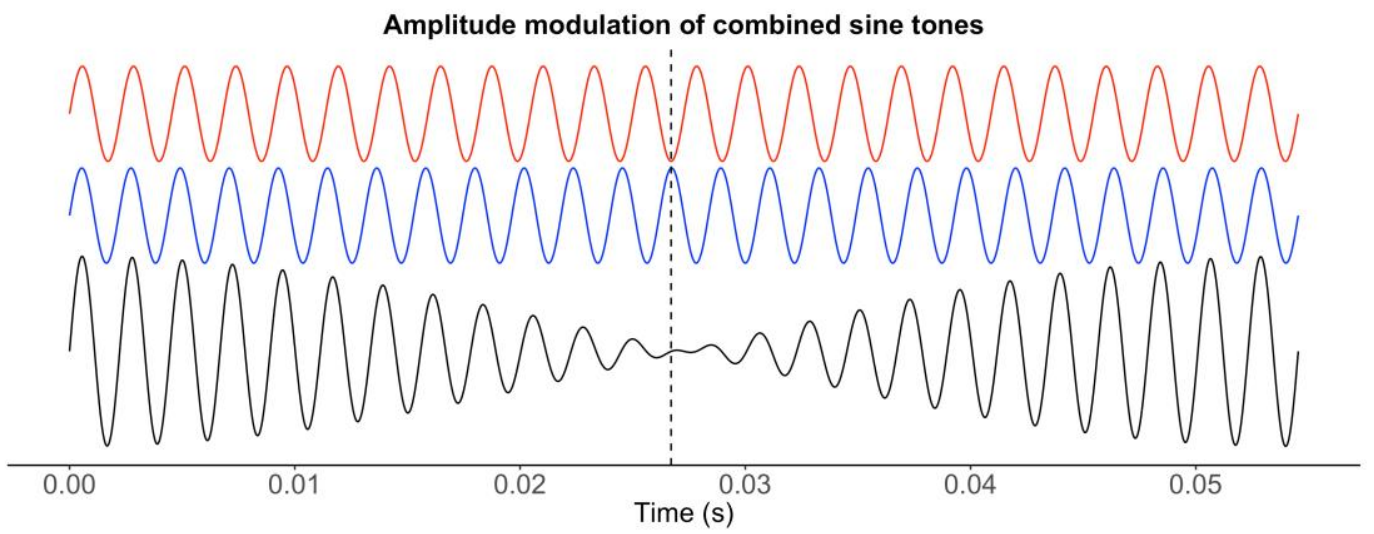

B

C

Spectra of complex tones at minor $2 \mathrm{nd}$ : No overlap of harmonic series

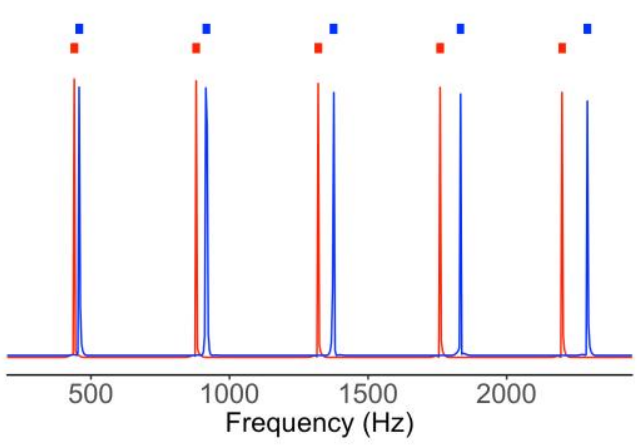

D

Waveforms of complex tones at minor $2 \mathrm{nd}$ : Combined amplitude beating

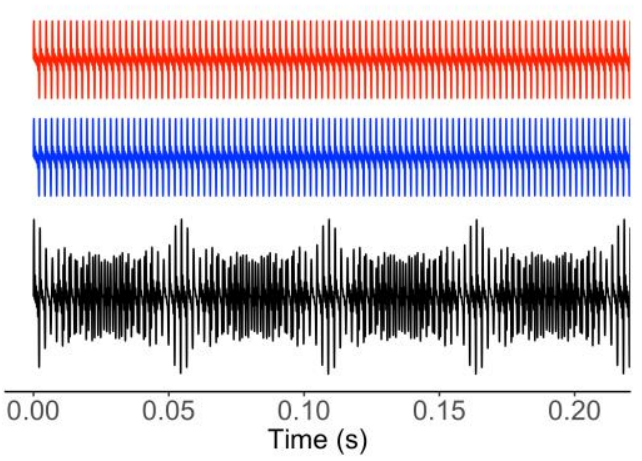

Spectra of complex tones at perfect 5th: Overlap of harmonic series

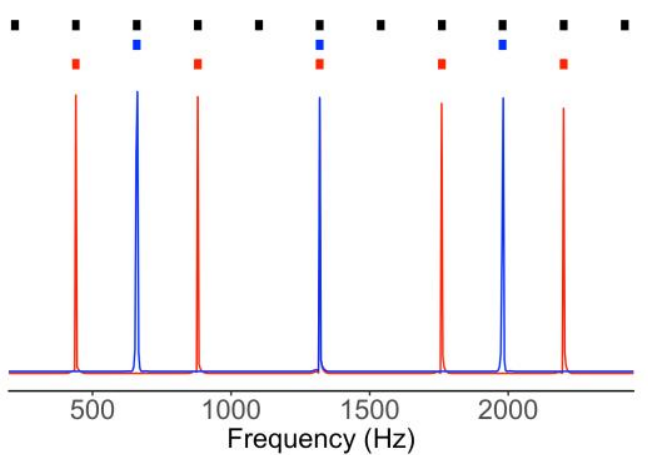

E

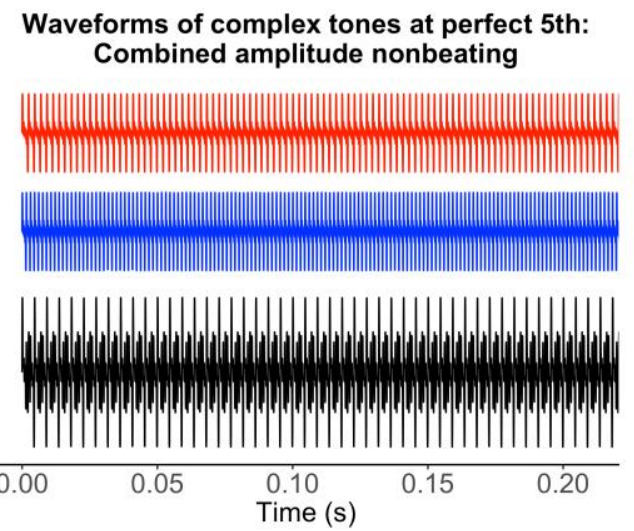

Figure 1. Examples of beating and harmonicity for dissonant (minor $2^{\text {nd }}$ ) and consonant (perfect $5^{\text {th }}$ ) intervals. Panel A: Combining the upper and middle sine tones results in the lower waveform, which has a fluctuating amplitude. Panels B and C: Complex tones have energy at a 
fundamental frequency and integer multiples of that frequency, as indicated by spikes of reducing amplitude. In the case of the dissonant interval (Panel B), the spacing between the combined peaks (red and blue bars) does not follow an evenly-spaced series. In the case of the consonant interval (Panel C), the spacing between the combined peaks (red and blue bars) overlaps considerably with a single, evenly-spaced harmonic series (black bars). Panels D and E: Combining complex waveforms at a dissonant interval (Panel D) leads to rapid amplitude fluctuations (i.e., beating / roughness), but amplitude does not fluctuate rapidly at a consonant interval (Panel E). 

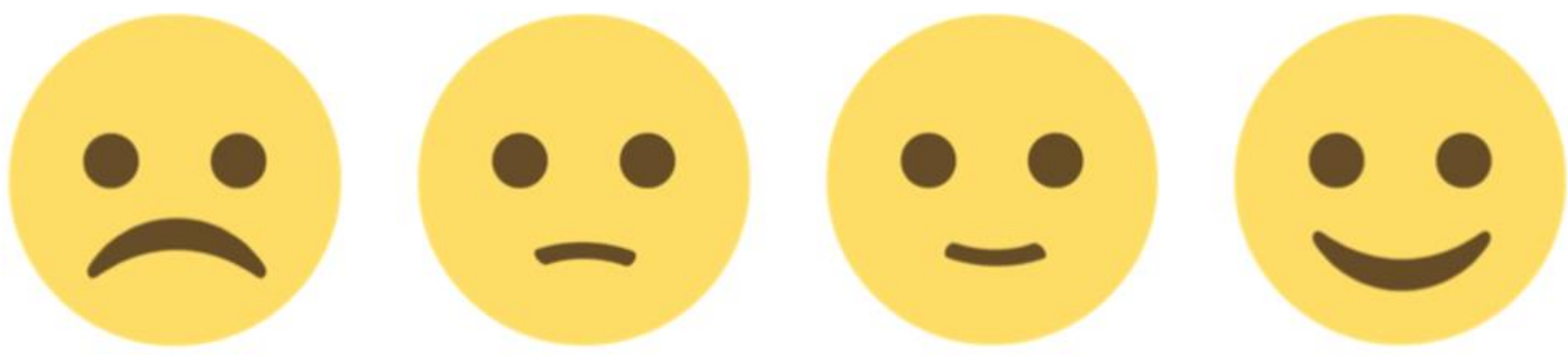

Figure 2. Visual response options. Responses were coded on a scale from 1-4. 
A

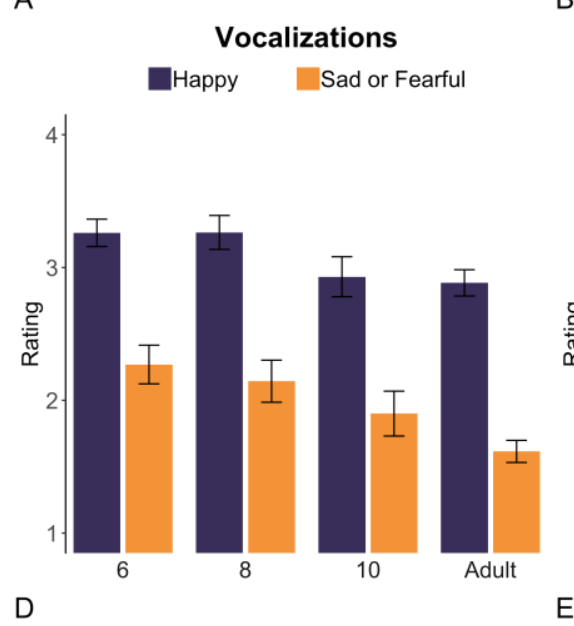

B
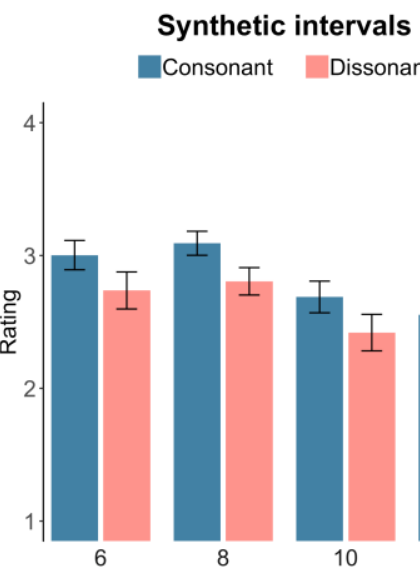

E
Consonant Dissonant

C

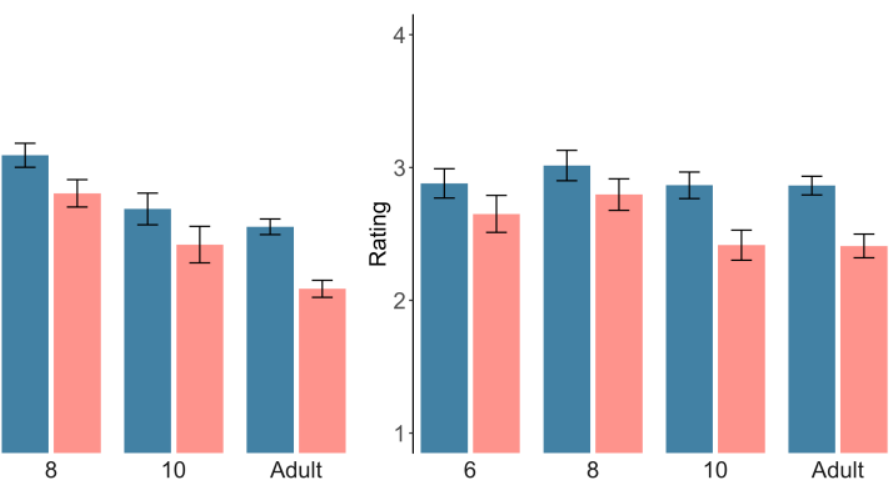

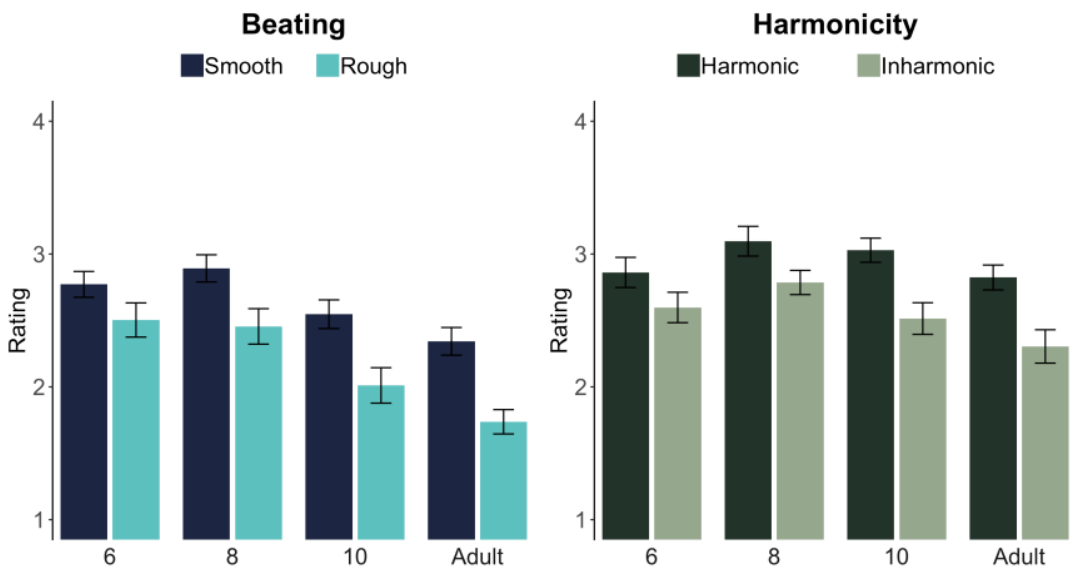

Figure 3. Mean ratings for stimuli across stimulus sets of Study 1 by age. Panel A visualizes ratings for emotional vocalizations. Panels $\mathrm{B}$ and $\mathrm{C}$ visualize ratings for consonant and dissonant intervals that differed in timbre (i.e., synthetic in B, sung in C). Panels D and E visualize ratings from the harmonicity and beating stimulus sets, separately for each type of trial (see Stimuli). Error bars are standard error of the mean. 

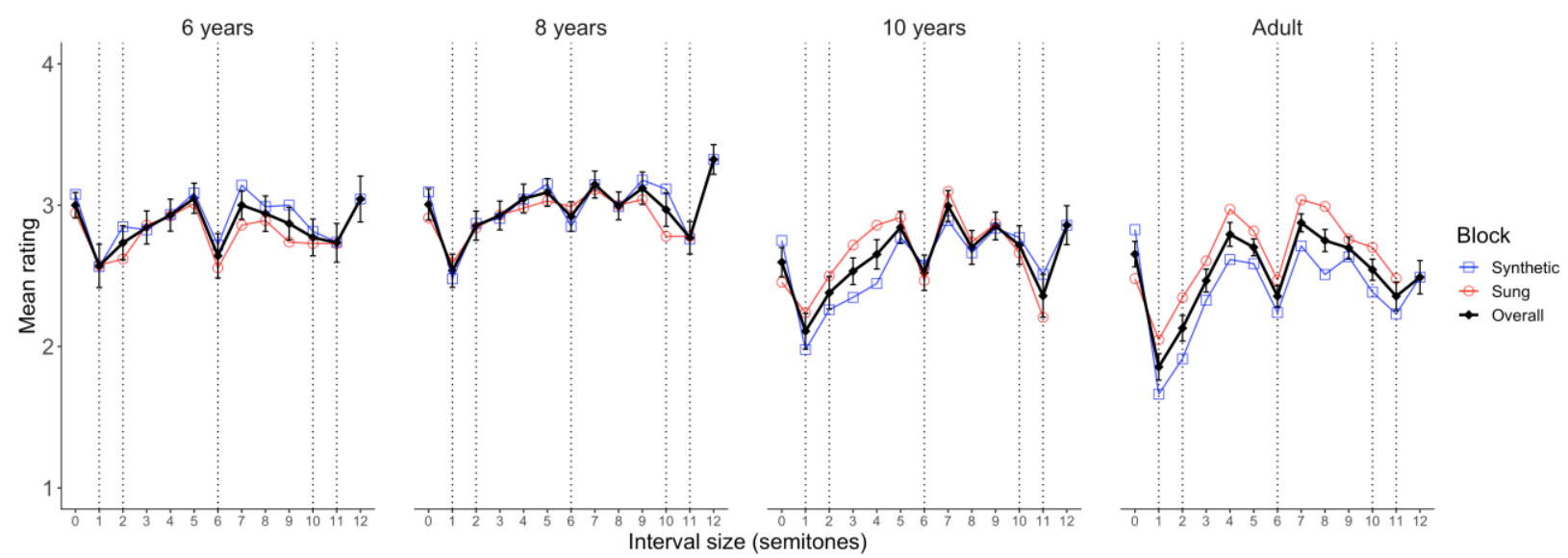

Figure 4. Ratings by interval and stimulus set for each age group in Study 1 . The blue line represents the synthetic set (unison to octave), the red line represents the sung set (unison to interval 11), and the black line represents the average across stimulus sets for each interval. Dashed vertical lines mark the dissonant intervals, as defined in McDermott et al. (2010). Error bars are standard error of the mean. 


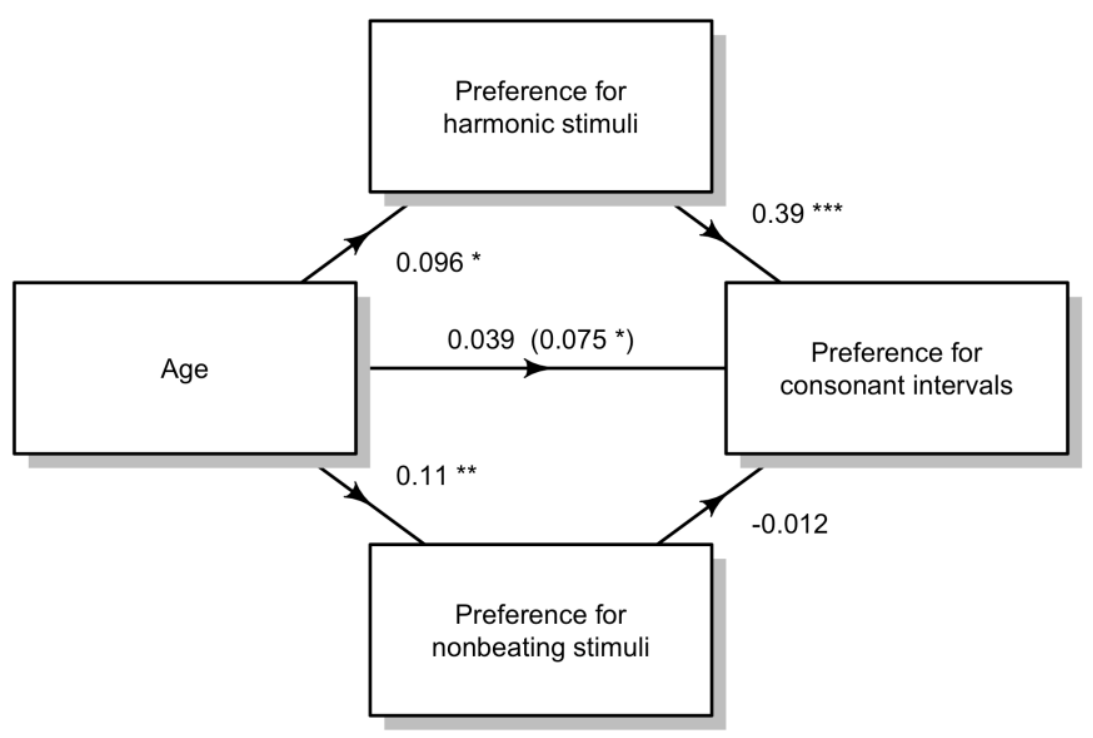

Figure 5. Beta estimates and significance $(* p<.05, * * p<.01, * * * p<.001)$ from the multiple mediation analysis in Study 1 (nonmusicians). The relationship between age and preference for consonance was fully mediated by acoustic preferences, specifically preference for harmonicity. The total effect of age (i.e., without mediators) is listed in parentheses. 
A

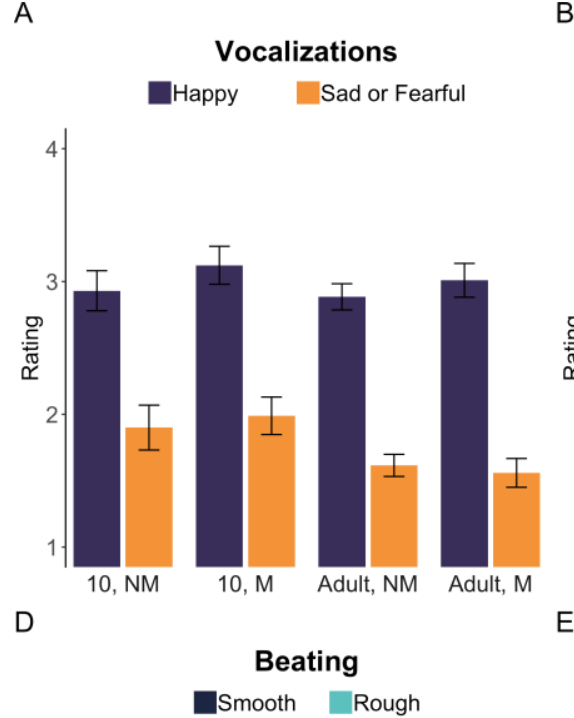

B

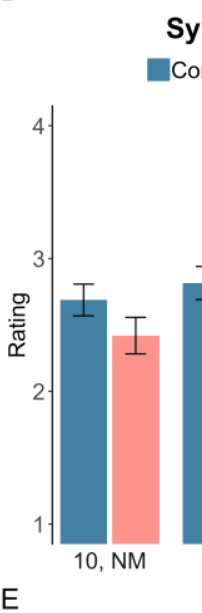

Synthetic intervals

Consonant Dissonant

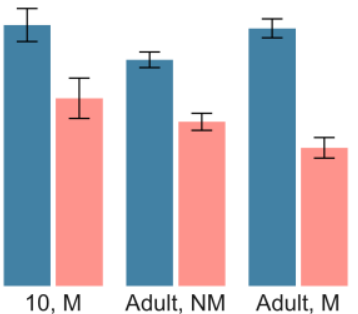

Harmonicity

DHarmonic Inharmonic
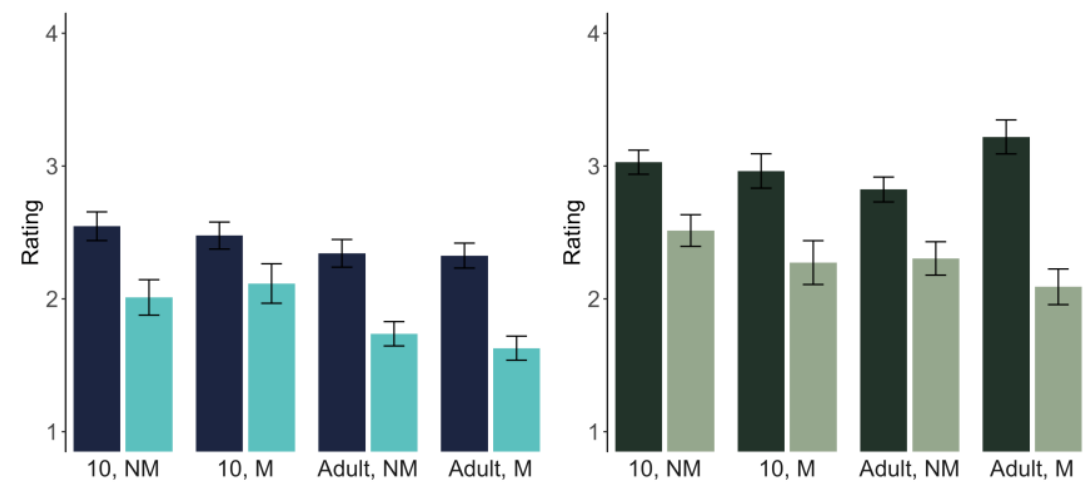

C Sung intervals

Consonant Dissonant

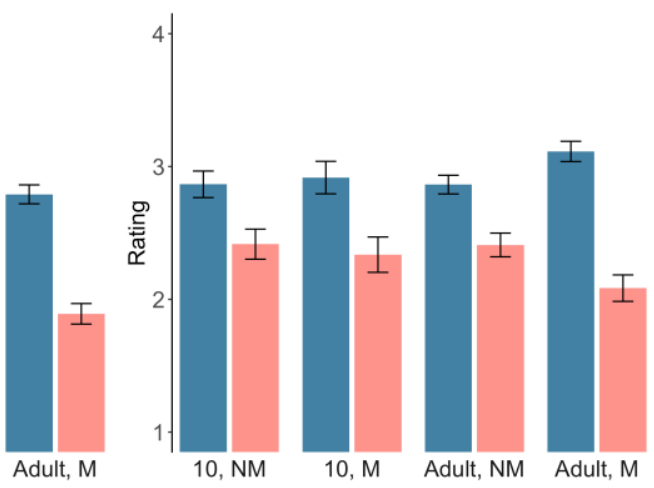

Figure 6. Mean ratings for stimuli across stimulus sets of Study 2 by age and musicianship (NM = nonmusicians, $\mathrm{M}=$ musicians). Nonmusician data was taken from Study 1. Panel A visualizes ratings for emotional vocalizations. Panels $\mathrm{B}$ and $\mathrm{C}$ visualize ratings for consonant and dissonant intervals that differed in timbre (i.e., synthetic in B, sung in C). Panels D and E visualize ratings from the harmonicity and beating stimulus sets, separately for each type of trial (see Stimuli). Error bars are standard error of the mean. 

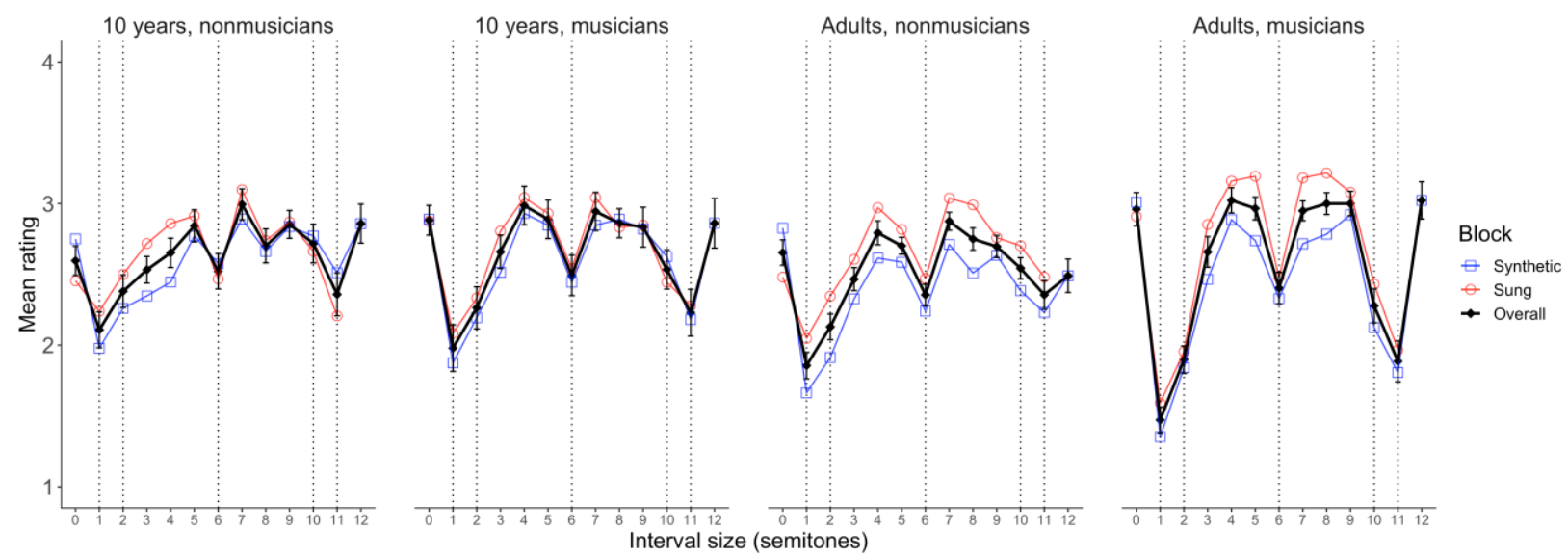

Figure 7. Ratings by interval and stimulus set for each age and musicianship group in Study 2.

Nonmusicians were included from Study 1. The blue line represents the synthetic set (unison to octave), the red line represents the sung set (unison to interval 11), and the black line represents the average across stimulus sets for each interval. Dashed vertical lines mark the dissonant intervals, as defined in McDermott et al. (2010). Error bars are standard error of the mean. 
A

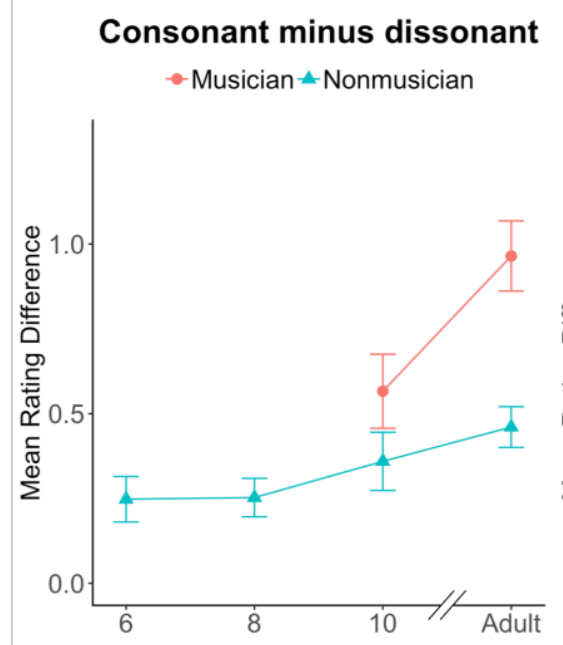

B Nonbeating minus beating

-Musician-Nonmusician

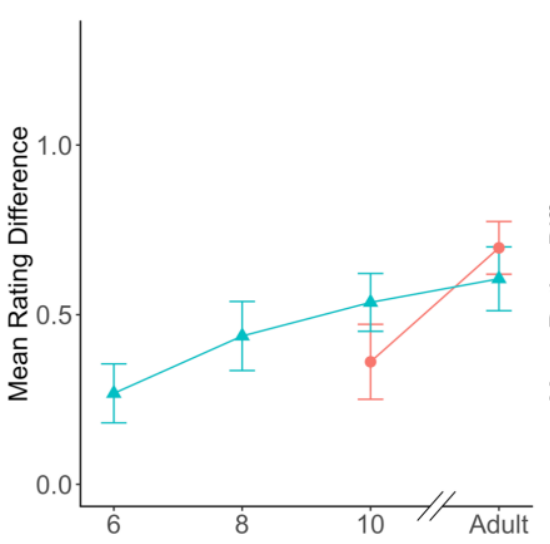

C Harmonic minus inharmonic -Musician-Nonmusician

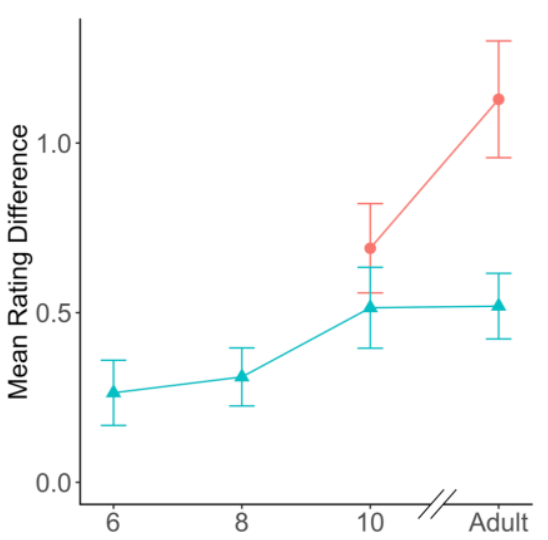

Figure 8. Preferences in Studies 1 and 2 by age and musicianship. Panel A displays the consonance advantage, or mean rating for consonant trials minus mean rating for dissonant trials. Ratings were collapsed across stimulus set averages (synthetic, sung). Panel B displays preference for non-beating stimuli. Panel C displays preference for harmonic stimuli. Error bars are standard error of the mean. 


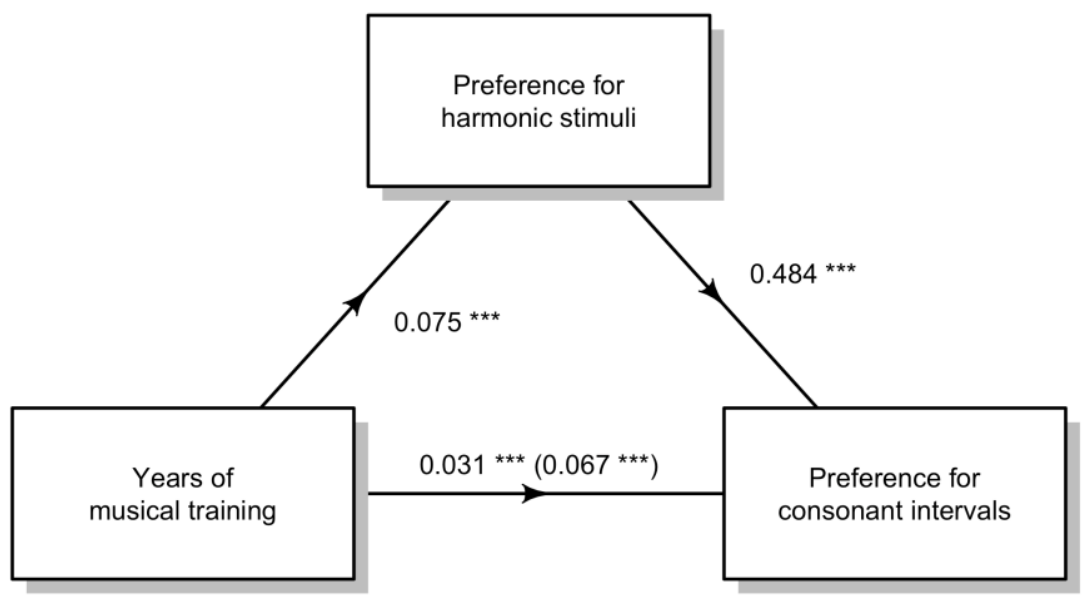

Figure 9. Beta estimates and significance $(* p<.05, * * p<.01, * * * p<.001)$ from the mediation analysis in Study 2. The relationship between musical training and preference for consonance was partially mediated by preference for harmonicity. The total effect of musical training (i.e., without the mediator) is listed in parentheses. 